Portland State University

PDXScholar

\title{
Ignoring Interprocessor Communication During Scheduling
}

Chintamani M. Patwardhan

Portland State University

Follow this and additional works at: https://pdxscholar.library.pdx.edu/open_access_etds

Part of the Computer Engineering Commons, and the Electrical and Computer Engineering Commons Let us know how access to this document benefits you.

\section{Recommended Citation}

Patwardhan, Chintamani M., "Ignoring Interprocessor Communication During Scheduling" (1992). Dissertations and Theses. Paper 4422.

https://doi.org/10.15760/etd.6300

This Thesis is brought to you for free and open access. It has been accepted for inclusion in Dissertations and Theses by an authorized administrator of PDXScholar. Please contact us if we can make this document more accessible: pdxscholar@pdx.edu. 


\title{
IGNORING INTERPROCESSOR COMMUNICATION DURING SCHEDULING
}

\author{
by \\ CHINTAMANI M. PATWARDHAN
}

A thesis submitted in partial fulfillment of the requirements for the degree of

\section{MASTER OF SCIENCE \\ in \\ ELECTRICAL AND COMPUTER ENGINEERING}

Portland State University

1992 
AN ABSTRACT OF THE THESIS OF Chintamani M. Patwardhan for the Master of Science in Electrical and Computer Engineering presented December 13, 1991.

Title: Ignoring Interprocessor Communication During Scheduling.

APPROVED BY THE MEMBERS OF THE THESIS COMMITTEE:
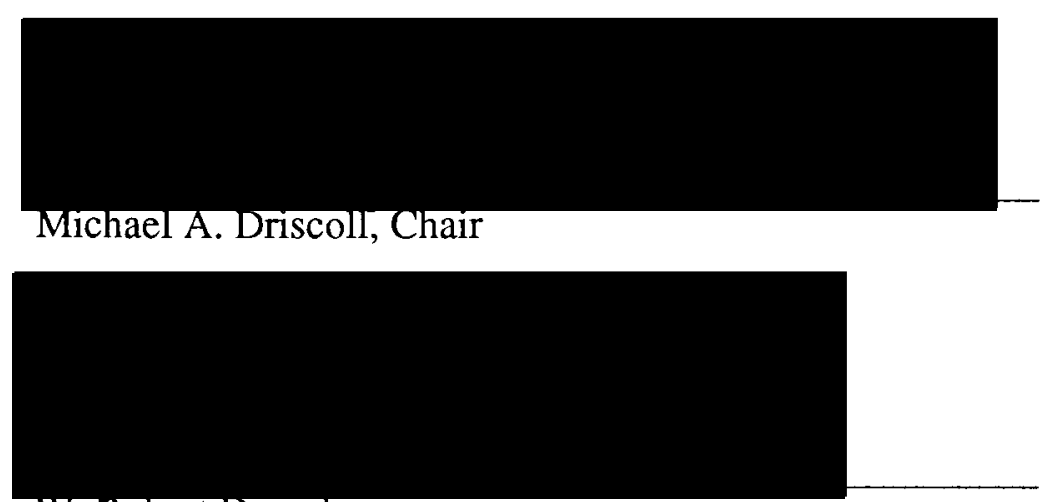

W. Robert Daasch

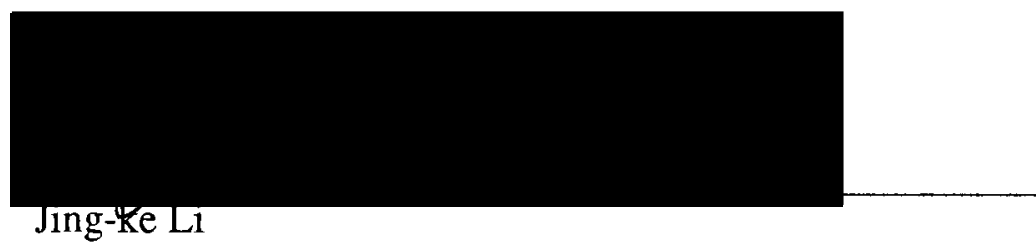

The goal of parallel processing is to achieve high speed computing by partitioning a program into concurrent parts, assigning them in an efficient way to the available processors, scheduling the program and then executing the concurrent parts simultaneously. In the past researchers have combined the allocation of tasks in a program and scheduling of those tasks into one operation. We define scheduling as a process of efficiently assigning priorities to the already allocated tasks in a program. Assignment of priorities is important in cases when more than one task at a processor is ready for execution. Most heuristics for scheduling consider certain parameters of the architec- 
ture and the program. These parameters could be the execution time of each operation in a program, the number of processors, etc. The impact of ignoring interprocessor communication (IPC) when ordering parallel tasks has, however, not been well studied.

We develop a model of the impact of ignoring IPC for parallel programs using barrier synchronization, when scheduled by a critical path algorithm. The model allows us to prove a theorem that identifies the cases for which IPC is important. For those cases, our model and experiments show that a program scheduled ignoring IPC performs almost as well as a program scheduled using IPC. Since including interprocessor communication in scheduling is expensive, we conclude that, for our programs and scheduling algorithm, it is reasonable to ignore IPC while scheduling. 


\section{TO THE OFFICE OF GRADUATE STUDIES:}

The members of the Committee approve the thesis of Chintamani M. Patwardhan presented December 13, 1991.

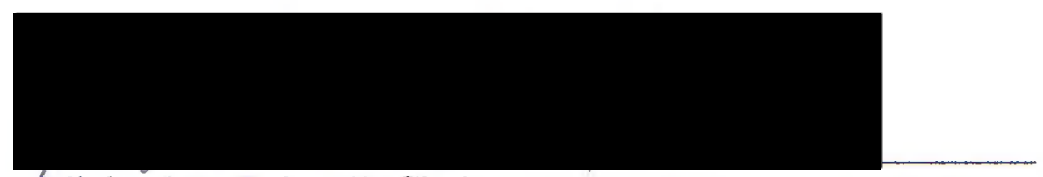

Michael A. Driscoll, Chair

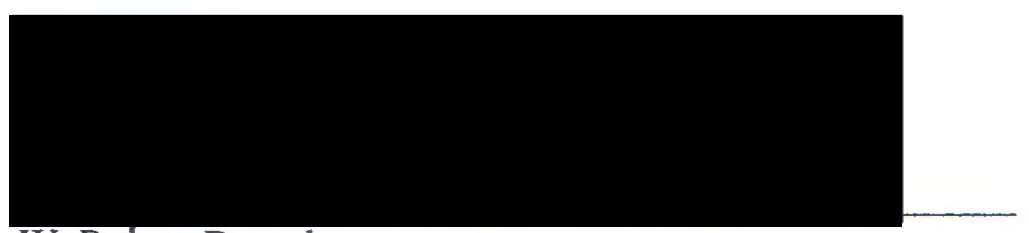

W. Robert Daasch

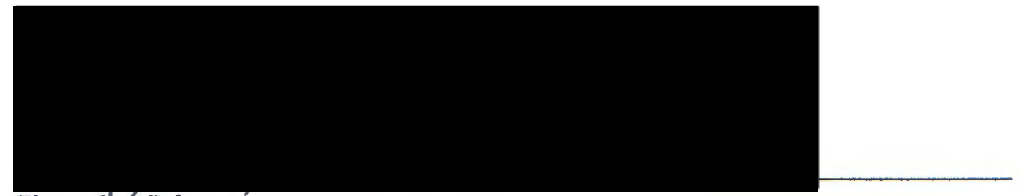

Jing-ké Li

APPROVED:

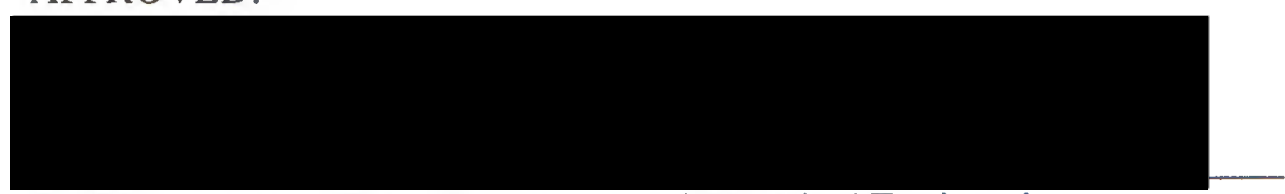

Rolf Schaumann, Chair, Department of Electrical Engineering

C. William Savery, vice Provost for Graduate Studies and Research 


\section{ACKNOWLEDGEMENTS}

Researching and writing a MS thesis is a lengthy process. During my academic life at PSU many people have provided assistance through financial and moral support. The most significant person, of course, has been my advisor, Dr. Michael Driscoll. I am grateful to him for giving me advice on courses, thesis topics, research pointers and in writing this document.

I would also like to thank other members of the ParPlum project: Jingsong Fu, De-Zheng Tang, Kiswanto Thayib, Satish Pai and Liono Setiowijoso for their support, co-operation and the hilarious moments we shared together.

I wish to thank the School of Business Administration and Dr. Beverley Fuller for the financial support.

Finally, thanks to my mother Mrs. Sheelaprabha Patwardhan and my brother Dr. Gagan Patwardhan for their financial and moral support during my entire academic life. Without their encouragement and support I probably would have never made it. 


\section{TABLE OF CONTENTS}

PAGE

ACKNOWLEDGEMENTS $\ldots \ldots \ldots \ldots \ldots \ldots \ldots \ldots \ldots \ldots \ldots \ldots \ldots \ldots \ldots$ iii

LIST OF TABLES $\ldots \ldots \ldots \ldots \ldots \ldots \ldots \ldots \ldots \ldots \ldots \ldots \ldots \ldots \ldots \ldots \ldots \ldots \ldots$

LIST OF FIGURES $\ldots \ldots \ldots \ldots \ldots \ldots \ldots \ldots \ldots \ldots \ldots \ldots \ldots \ldots \ldots \ldots \ldots$ vii

NOTATIONS AND GLOSSARY $\ldots \ldots \ldots \ldots \ldots \ldots \ldots \ldots \ldots \ldots \ldots \ldots \ldots \ldots$ ix

\section{CHAPTER}

I INTRODUCTION $\ldots \ldots \ldots \ldots \ldots \ldots \ldots \ldots \ldots \ldots \ldots \ldots \ldots \ldots$

1.1 Problem Statement $\ldots \ldots \ldots \ldots \ldots \ldots \ldots \ldots \ldots \ldots, 1$

1.2 Motivation and Purpose of this Research ........... 2

1.3 Thesis Overview $\ldots \ldots \ldots \ldots \ldots \ldots \ldots \ldots \ldots \ldots \ldots$

II OVERVIEW OF SCHEDULING METHODS . . . . . . . . 5

2.1 Background $\ldots \ldots \ldots \ldots \ldots \ldots \ldots \ldots \ldots \ldots \ldots \ldots \ldots \ldots \ldots \ldots$

2.2 Classification of Scheduling Algorithms .......... 6

2.3 Scheduling Algorithms and their Properties ......... 8

2.4 Scheduling Definition Function and Goal .......... 11

III PARALLEL PROGRAMS AND THEIR INFLUENCE ON GLOBAL SCHEDULES $\ldots \ldots \ldots \ldots \ldots \ldots \ldots \ldots \ldots \ldots$

3.1 Importance of Parallel Paths for Global Schedule ..... 16

3.2 Suitable Scheduling Algorithms . ............. 19

3.3 Importance of A Cut in A Path .............. 20

3.4 Experimental Focus .................... 25

IV MODELING SCHEDULE EXECUTION TIMES .......... 26 
4.1 An Ideal Graph . . . . . . . 26

4.2 Modeling Approach . . . . . . . . 27

4.3 Graph Behavior versus Variations in Key Parameters . . . $\quad 30$

4.4 Behavioral Prediction of Experimental Outcome . . . . . . 36

V EXPERIMENTAL RESULTS AND THEIR ANALYSIS . . . . . 37

5.1 Variation in Paths 1 and 2 ( Path 3 Constant )....... 37

5.2 Variation in Path 3 (Path 1 and 2 Constant )....... 42

5.3 Variation in Path 1 ( Path 2 and 3 Constant ) ........ 46

5.4 Variation in Path 2 ( Path 1 and 3 Constant) ....... 49

5.5 Model Performance Analysis . . . . . . . . . . 52

VI CONCLUSION $\ldots \ldots \ldots \ldots \ldots \ldots \ldots \ldots \ldots \ldots \ldots \ldots$

6.1 Significance of this Research ............. 53

6.2 Future Work................... 54

REFERENCES ............................. 57 
I Schedule for Graph in Figure $8 \ldots \ldots \ldots \ldots \ldots \ldots \ldots \ldots \ldots \ldots \ldots \ldots$

II Execution Time for Graphs with Path 1 and 2 Varying Simultaneously . . 39

III Execution Time for Graphs with Path 3 Varying (Global Schedule) ... 43

IV Execution Time for Graphs with Path 3 Varying (Local Schedule) . . . . 44

V Execution Time for Graphs with Path 1 Varying (Global Schedule) ... . 47

VI Execution Time for Graphs with Path 1 Varying (Local Schedule) . . . . 47

VII Execution Time for Graphs with Path 2 Varying (Global Schedule) ... . 50

VIII Execution Time for Graphs with Path 2 Varying (Local Schedule) . . . . . 50

IX Schedule for Fine Grain $\ldots \ldots \ldots \ldots \ldots \ldots \ldots \ldots \ldots \ldots \ldots \ldots \ldots \ldots \ldots \ldots \ldots$ 


\section{LIST OF FIGURES}

1 A Graph depicting the Importance of Scheduling

2 Assignment of Priorities When Graph in Figure 1 is

Scheduled Globally and Locally . . . . . . . . . . . . . 14

$3 \quad$ A General Parallel Program $\ldots \ldots \ldots \ldots \ldots \ldots \ldots \ldots \ldots \ldots \ldots$

4 A Parallel Program with Paths Assigned to Processors . . . . . . . . . . 18

5 Firing Sequence of Paths in Figure 4 When Scheduled

by Granski's Heuristic ....................... 20

6 Graph with Uncut Parallel Strings Allocated to two Processors . . . . . . . 21

7 Mapping Pieces of a Cut Path to different Processors . . . . . . . . . . . . 22

8 An Ideal Graph for Modeling Execution time of

Global and Local Schedules ........................ 26

9 Dataflow Graph in Figure 8 Modeled as a Set of Paths

between Fork and Join Points . . . . . . . . . . . . . . 27

10 Execution Sequence of nodes in Figure 8 for Global Schedule . . . . . . . . 29

11 Execution Sequence of nodes in Figure 8 for Local Schedule . . . . . . . . 29

12 General Execution Sequence for Globally Scheduled Graph in Figure 8 . . $\quad 31$

13 General Execution Sequence for Locally Scheduled Graph in Figure $8 \ldots \ldots \quad 31$

14 Graph of Variation in number of Nodes in Path 1 and 2

Versus total Execution time of Globally and Locally Scheduled graph . . . $\quad 40$

15 Graph of Variation in Number of Nodes in Path 1 and 2

Versus Difference in total Execution time ................ 40 
16 Child Execution time for Globally and Locally Scheduled

Graph in Figure $8 \ldots \ldots \ldots \ldots \ldots \ldots \ldots \ldots \ldots \ldots \ldots \ldots \ldots \ldots \ldots \ldots$

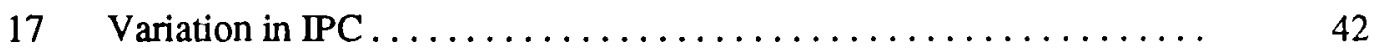

18 Graph for Variation in Number of Nodes on Path 3

Nodes on Paths $1 \& 2=200 \ldots \ldots \ldots \ldots \ldots \ldots \ldots \ldots \ldots \ldots \ldots$

19 Graph for Variation in Number of Nodes on Path 3

Nodes on Paths $1 \& 2=100 \ldots \ldots \ldots \ldots \ldots \ldots \ldots \ldots \ldots \ldots \ldots$

20 Graph for Variation in Number of Nodes on Path 3

Nodes on Paths $1 \& 2=50 \ldots \ldots \ldots \ldots \ldots \ldots \ldots \ldots \ldots \ldots$

21 Graph for Variation in Number of Nodes on Path 1

Nodes on Paths $2 \& 3=10 \ldots \ldots \ldots \ldots \ldots \ldots \ldots \ldots \ldots$

22 Graph for Variation in Number of Nodes on Path 1

Nodes on Path $2=50 \&$ Path $3=10 \ldots \ldots \ldots \ldots \ldots \ldots \ldots \ldots \ldots$

23 Graph for Variation in Number of Nodes on Path 1

Nodes on Path $2=100 \&$ Path $3=10 \ldots \ldots \ldots \ldots \ldots \ldots \ldots . \ldots . \ldots . \ldots$

24 Graph for Variation in Number of Nodes on Path 2

Nodes on Path $1=50 \&$ Path $3=10 \ldots \ldots \ldots \ldots \ldots \ldots \ldots \ldots \ldots \ldots$

25 Graph for Variation in Number of Nodes on Path 2

Nodes on Path $1=100 \&$ Path $3=10 \ldots \ldots \ldots \ldots \ldots \ldots \ldots \ldots \ldots$

26 A Comparison of Global and Local Scheduler Execution times ........ 52

27 An example of Fine and Coarse Grain Scheduling $\ldots \ldots \ldots \ldots \ldots \ldots$ 


\section{NOTATIONS AND GLOSSARY}

$\tau_{g}$

$\tau_{l}$

$m_{i}$

$P \dot{k}, j$

$T \dot{k}, j$

$W^{i, j}$

$C^{j, k}$

$W_{g}^{i, j}$

$W i, j$

$t^{i, j, k}$

$c_{i, j}^{k}$
Execution time of globally scheduled graph

Execution time of locally scheduled graph

Number of pieces of path $\mathrm{i}$

$j$ ' th piece of path $i$ assigned to processor $\mathrm{k}$

Time to execute $P^{i, j}$ on processor k

Weight of string $P^{i, j}$

Communication time between strings $P^{i, j}$ and $P^{i, k}$

Weight of string $P^{i, j}$ when scheduled globally

Weight of string $P^{i, j}$ when scheduled locally

Overlap between execution of nodes $i, j$ and $k$

Overlap of communication between nodes $\mathrm{i}, \mathrm{j}$ and the execution of node $k$ 


\section{CHAPTER I}

\section{INTRODUCTION}

\subsection{PROBLEM STATEMENT}

With the advent of multiprocessor architectures came the task of efficiently processing a set of parallel tasks. A goal of parallel processing is to achieve very-high speed computing by partitioning a sequential program into concurrent parts, assigning them in an efficient way to the available processors, scheduling the program and then executing the concurrent parts, simultaneously. This procedure is thus comprised of three steps : partitioning, allocation and scheduling [10]. Partitioning, allocation and scheduling are multiprocessor dependent issues. Partitioning is necessary to ensure that the granularity of the parallel program is coarse enough for the target multiprocessor architecture, without losing too much parallelism. Scheduling, on the other hand, can improve the response time of a multiprocessor architecture by achieving a good processor utilization. However, to do this, tasks should be assigned to individual processors in a way that exploits available parallelism. Finding optimal schedules for precedence-related tasks on a multiprocessor system is an extensively studied problem and has been found to be NPhard for most practical problems [11], [4].

Most of the research done to date for efficient scheduling does not consider interprocessor communication overhead such as processor communication. Such an assumption is a reasonable approximation to some real multiprocessor systems, however it is no longer valid for message passing multiprocessors or computer networks. For such sys- 
tems interprocessor communication overhead is clearly an important aspect of performance and cannot be ignored. With unit-time tasks and tree precedences the scheduling problem is known to be solvable in polynomial time [15] but with the introduction of communication delays because of message passing between processors, this case may again become NP-hard.

\subsection{MOTIVATION AND PURPOSE OF THIS RESEARCH}

Although several scheduling algorithms have been proposed by researchers, it is important that the scheduling algorithm used executes reasonably quickly and that its execution time increases slowly as the size of the job being scheduled increases [5]. In other words, the scheduling algorithm should be efficient. Hwang [16] has shown that interprocessor communication (IPC) is an important parameter to be considered while scheduling and cannot be ignored. Hwang and other researchers have considered the process of allocating tasks to individual processors to be the same as scheduling. El-Rewini [9] has shown that allocation is sensitive to IPC which justifies Hwang's case. We, on the other hand, consider allocation and scheduling as two distinct processes. Allocation, in our sense, is a process of allocating tasks to individual processors, whereas scheduling is a process of efficiently assigning priorities to the already allocated tasks. Scheduling, therefore, in our case may ignore IPC. The research problem in this thesis is to explore the advantages and disadvantages in ordering parts of a program assigned to processors while considering and ignoring the communication overhead. The motivation behind this research stems from the idea that estimation of interprocessor communication may prove to be very expensive, especially when the job to be scheduled is small and the algorithm takes a long time to schedule it. In addition, the resulting schedule may be no different from the schedule that could be obtained by neglecting the IPC. We would therefore not attain minimum execution time, which is a key parameter to take advantage of parallel 
processing.

Since many algorithms have been suggested by various researchers for efficient scheduling, we will focus on the following objectives in this thesis:

1. Identifying the scheduling algorithms suitable for our research.

2. Identifying graphs and their properties that show a difference in assignment of priorities and execution time while considering and ignoring the IPC.

3. Designing a simple dataflow graph and models predicting its performance under the situation cited above.

4. Experimental testing of the model graph on the ParPlum interpreter [10].

5. Evaluating the results obtained from experiments.

It is our hypothesis that for some programs and scheduling algorithms, operations at a processor can be ordered without regard to the interprocessor communication time.

\subsection{THESIS OVERVIEW}

Chapter II begins with an overview of research carried out for efficient scheduling of graphs, the definitions of global and local scheduling, and their importance. Chapter III tries to find the properties of mappings that show a difference in global and local schedules. To do so, it presents possible mappings of a graph on an architecture and the effect of global and local schedules on the execution time of such mappings . Chapter IV begins by showing a graph designed for experiments, which has the properties needed to see a difference in assignment of priorities using global and local schedules and later presents models that can predict the performance of the experimental graph. Chapter $\mathrm{V}$ presents the results of executing the model graph on the ParPlum interpreter after applying the global and local scheduling techniques to it. A comparison of the experimental results with the results predicted by the models is also analyzed. Finally, Chapter VI 
presents conclusions, reviews the usefulness of the research presented herein, and points out directions for future research. 


\section{CHAPTER II}

\section{OVERVIEW OF SCHEDULING METHODS}

\subsection{BACKGROUND}

Processor scheduling has a long history of study. Early research on the scheduling problem concentrated on the scheduling of work in job shops. Later, researchers investigated the problem of scheduling jobs on uniprocessor systems using multitasking operating systems [33]. However, with the arrival of multiprocessing systems came the task of scheduling the jobs on them so as to minimize the parallel execution time of a job and to better utilize the processors in the system. An efficient scheduling algorithm should allocate a set of tasks to the available processors of a multiprocessor system and determine the sequence or order of execution of the tasks allocated to each member processor. Most of the scheduling problems suggested to date are NP-Hard [4], [11]. Some algorithms may usually have low computational requirements, but become enumerative under certain circumstances. Even most of the relaxed and simplified subproblems, derived from the original scheduling problems by adding restrictions to them, fall into the class of NP-Hard problems [4]. The restrictions usually added are :

1. Preemption not allowed;

2. The number of parallel processors used;

3. Attributes of tasks such as topology of the task graph; and

4. Uniformity of task processing times. 
Some scheduling problems can be solved in polynomial time [24]. For example, when the task graph is a tree and the execution time of all the tasks is one time unit, $\mathrm{Hu}$ [15] showed that by using a list scheduling algorithm an optimal solution can be found in $\mathrm{O}(\mathrm{n})$ time. Hu's algorithm assigns a level number to each node in the task graph on the basis of the length of the longest path from the node to an exit node of the precedence graph. In a second case when the precedence relation is arbitrary but the number of processors is limited to two, Coffman \& Graham [4] showed that by using a list scheduling algorithm similar to $\mathrm{Hu}$, a solution can be reached in $\mathrm{O}\left(n^{2}\right)$ time. The two special problems mentioned above, however, become NP-Hard [4] if any one restricting condition is relaxed. To circumvent these problems, heuristic algorithms have been proposed to obtain approximate or suboptimal solutions to such problems in polynomial time.

\subsection{CLASSIFICATION OF SCHEDULING ALGORITHMS}

While various scheduling problems have been studied for years, a detailed survey of earlier results presented by Gonzalez [11] describes a scheme by which the scheduling problems can be classified. His criteria takes into consideration the following:

1. The type of precedence among tasks in the program;

2. The type of processors used in the architecture; and

3. The number of processors in the architecture.

The tasks in the program may have arbitrary precedence or no precedence at all, the architecture may have any number of processors and the processors themselves could be homogeneous or heterogenous. Kruatrachue [22], on the other hand, suggests that the results in parallel processor scheduling can be classified into five classes on the basis of the goal of the scheduler, the type of task to be scheduled and the parallel processor system. The groups in turn would be as follows: 
1. Precedent scheduling: The goal of the scheduler here is to minimize the schedule length. The task is usually represented as an acyclic directed graph $\mathrm{G}(\mathrm{T},<)$ where $\mathrm{T}$ is a set of nodes representing tasks $\left\{T_{1}, T_{2}, T_{3}, \ldots ., T_{n}\right\}$, and $<$ is a set of arcs between those nodes representing precedent order. $T_{i}<T_{j}$ means that $T_{i}$ can be executed only after $T_{j}$ has completed its execution. The communication time between any two tasks is assumed to be zero. Hu's list scheduling algorithm and Coffman \& Graham's algorithm fall in this category. A less complex algorithm than Coffman's is suggested by Sethi [33]. It provides the same schedule but in $\mathrm{O}(\mathrm{na}(\mathrm{n})+\mathrm{e})$ time units, where $\mathrm{e}$ is the number of edges in the graph, and $\mathrm{a}(\mathrm{n})$ is a cost function of $n$. Kaufman [20] has suggested an algorithm which is applicable to tree graphs with nodes having arbitrary execution times. The time complexity of this algorithm is $1+\frac{(p-1) t}{T}$, where $t$ is the longest path execution time, $\mathrm{T}$ is the summation of execution times of all nodes in the graph and $\mathrm{p}$ is the number of processors.

2. Communication precedent scheduling: The job of the scheduler is similar to that described in precedent scheduling with the addition that the scheduler considers communication delays between different processors. $\mathrm{Yu}$ [Yu 84] has suggested a heuristic algorithm that considers communication delays while assigning tasks to processors, assuming the communication delays between processors to be identical. For large communication delays, results obtained for Yu's algorithm are better than Hu's algorithm.

3. Load balancing communication scheduling: The goal of the scheduler is to balance the load among the processors and to minimize the communication delay. Since this type of objective is different from the scheduling with which we are concerned, we will not go into details of this group.

4. Dynamic task scheduling: The goal of the scheduler is again to minimize the 
schedule length. Here, however, the node execution times, the communication time between processors, the number of nodes in the graph and the precedent constraint are varying and can be changed during execution. Graphs with loop or branching statements usually fall into this category since the number of iterations of a loop may not be known beforehand. Ramamoorthy [30] and Kund [23] have proposed a heuristic for dynamic scheduling in which the schedule is determined at runtime. These complex schedule strategies however, introduce excessive overhead in this case and there is no guarantee of near optimal schedules.

5. Independent task scheduling: The goal of the scheduler is to improve the load balancing and meeting the individual task deadlines.

According to Coffman [4], scheduling algorithms can be classified as follows:

1. Static vs Dynamic - Static algorithms schedule the whole graph only once. Dynamic algorithms on the other hand can reschedule tasks that are being executed.

2. Deterministic vs. Stochastic - Deterministic algorithms require prior knowledge of the execution times of nodes in a task graph and the communication time between them. Stochastic algorithms use probabilistic models to calculate the execution time of the nodes and the communication times between them.

3. Preemptive vs. Non-preemptive - Preemptive algorithms handle tasks which can be stopped during execution and resume execution at a later time. Nonpreemptive algorithms, on the other hand, cannot interrupt the execution of tasks until they finish their execution.

\subsection{SCHEDULING ALGORITHMS AND THEIR PROPERTIES}

The scheduling problem studied so far by researchers is to assign tasks in a partitioned program to processors, to minimize the execution time. The execution time in turn 
depends on processor utilization and on the overhead incurred in interprocessor communication. From the theoretical standpoint there are two types of scheduling disciplines 1) general and 2) list [25]. A general schedule usually describes the exact initiation time of the tasks and so it requires the execution time of the tasks to be deterministic. To ensure the shortest execution time of the schedule generated, some of the processors may remain idle, waiting for some important tasks to get ready for execution, even though some other tasks are already waiting to be executed [29]. While general scheduling has many shortcomings when applied in practice, where perfect synchronization is impossible, it still serves as the best one can do to optimize execution in a static sense. List scheduling, on the other hand, is simply a priority list of the tasks involved, and no processor is left idle during execution. The list schedule is an effective and realistic technique in practice and is applicable even if the task execution times vary greatly from one another [25]. Another important fact is that if the execution time of all the tasks are the same, then there is no distinction between an optimal list schedule and an optimal general schedule.

Since list scheduling is good in practice, we will now examine a couple of list scheduling algorithms that interest us. List schedules are a class of implementable schedules in which tasks are assigned priorities and placed in a list ordered by decreasing magnitude of priority. Whenever executable tasks contend for processors, the task that has the highest priority is assigned to the processor first. Various algorithms based on list scheduling algorithms have been suggested, some of the prominent among them being: 1) Critical path; 2) Critical path most immediate successor first; and 3) longest path scheduling algorithm.

Critical path scheduling is a type of list scheduling. It is nonpreemptive, static, deterministic and has been shown to achieve good results under most circumstances [1], [21]. In critical path scheduling the exact interval and the processor assigned to a given task need not be specified. It is used primarily for homogeneous architectures. 
The critical path most immediate successor first (CPMISF) heuristic was proposed by Kashara \& Narita [19] and is an improved version of the critical path method, with the following enhancement: tasks that do not belong to the critical path are given priority based on the number of their successors. In other words, the more successor nodes of a task, the higher the priority assigned. This approach has been reported to obtain optimal or close to optimal solutions for large scale problems with large numbers of tasks. The CPMISF method is a generalized form of Hu's algorithm. Hu's algorithm fails to produce the best schedule when the number of tasks at any one level is more than one. This problem is solved in the CPMISF method by assigning the highest priority to a task that has the largest number of immediate successive tasks. The procedure involved in the CPMISF method is as follows:

1. Determine the level $l_{i}$ for each task.

2. Build a priority list in the descending order of $l_{i}$ and the number of immediately successive tasks.

3. Execute list scheduling on the basis of the priority list.

The longest path scheduling algorithm assigns those tasks that are farthest from the root of the tree at the time of assignment to any free processors. Hu [15] showed that when all tasks have the same execution times, the total execution time for the longest path algorithm is minimal or, in other words, no other nonpreemptive algorithm can give better result. The only drawback of this scheduling method, however, is that it is applicable to tree graphs and none other. In this thesis we have used the "critical path" algorithm widely. What makes the critical path algorithm so important to us is that it has been shown to achieve good results under most circumstances. Also, since no schedule for an arbitrary graph can be shorter than its critical path, the critical path is the key to successful precedence scheduling [22]. 
Most of the research done to date for efficient scheduling does not consider the interprocessor communication overhead, such as processor communication. Some researchers have argued that in the case of computer networks, the interprocessor communication time is significant and cannot be ignored [16]. Allocation has been shown to be sensitive to IPC [9], therefore the statement of those researchers holds true when allocation and scheduling are combined and treated as one process.

Rayward-Smith has presented a heuristic known as generalized list scheduling [31] for the above mentioned problem, with unit communication times (UCT) and unit execution (UET) times as the restrictions. The heuristic follows the same greedy strategy as in Graham's list scheduling [12] in which no processor is idle if there exists some task that it could process. A task $\mathrm{T}$ can be processed on a processor $P_{i}$ at time $\tau$ if $\mathrm{T}$ has no immediate predecessor, or each predecessor of T has been scheduled on processor $P_{i}$ at time less than or equal to $\tau-1$ or on a processor $P_{j} \neq P_{i}$ at time less than or equal to $\tau-2$. Hwang, Chow and Anger [16] refined Rayward-Smith's heuristic to present a new heuristic called Earliest Task First (ETF), which reduced the time complexity and has an improved performance bound.

\subsection{SCHEDULING DEFINITION FUNCTION AND GOAL}

In parallel processing, the given task must be mapped onto a given parallel architecture. This usually means partitioning the given task into subtasks, allocating those subtasks onto the available processors and then scheduling the subtasks on each individual processor [14]. Most researchers in the past have addressed the issue of allocation of subtasks and scheduling of those individual subtasks as one. To simplify the mapping process we assume that allocation and scheduling are two distinct steps. Allocation concentrates on allocating subtasks to processors so as to minimize the interprocessor communication time and on other issues such as load-balancing. Scheduling, on the other hand, 
concentrates on an efficient assignment of priorities to the operations in the subtasks to minimize the execution time of the program. Scheduling, or assignment of priorities, is important in such cases when more than one of the nodes in a subtask at a processor are ready for execution. For example, nodes 1 and 2 in Figure 1 are both ready for execution and, further, some nodes assigned to processor 2 are dependent on node 2 . If node 1 and its successors are executed before node 2 , then processor 2 will remain idle while waiting for a result to arrive from node 2 , which would result in an increase in total execution time due to underutilization of resources. On the contrary, if node 2 is executed first, then processor 2 could start execution of the nodes assigned to it sooner than in the previous case and the overall execution time of the subgraph would be reduced considerably.

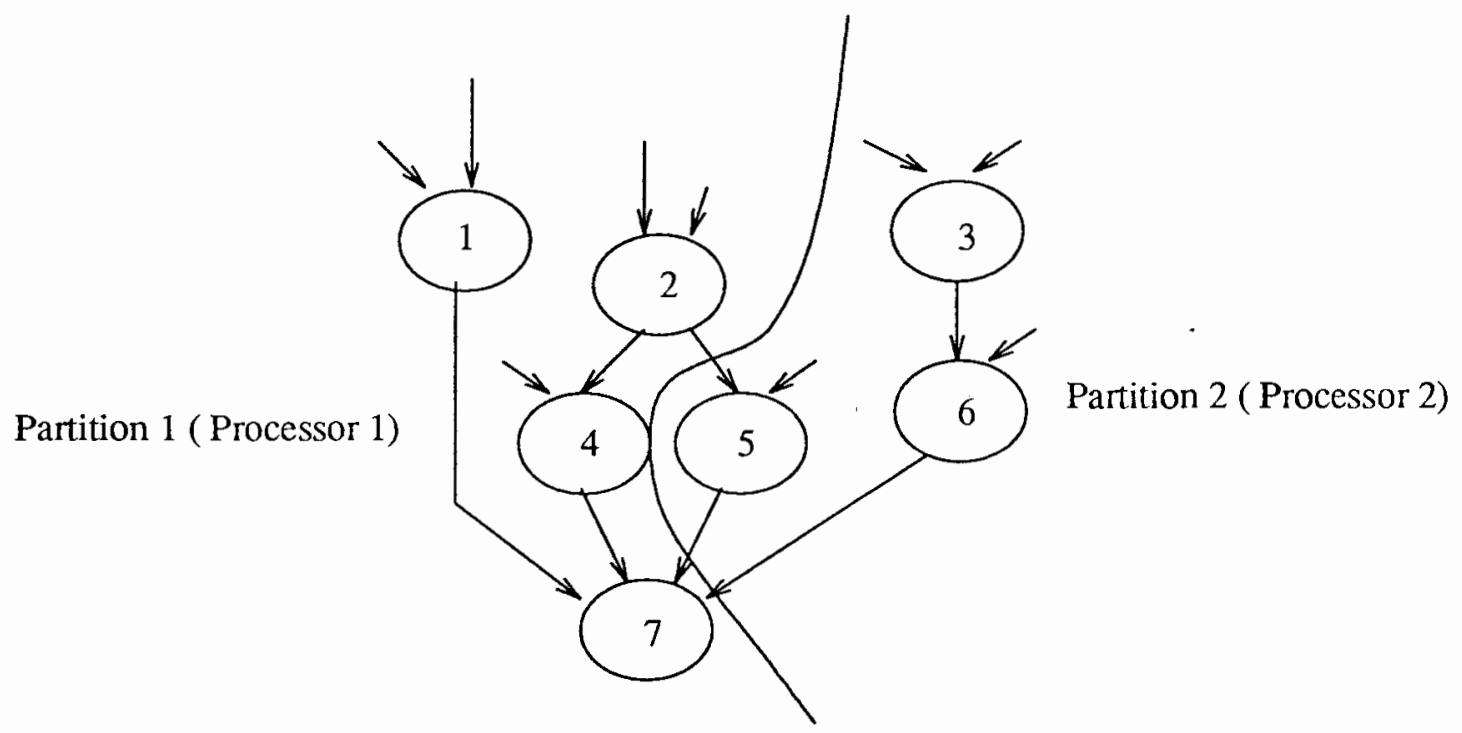

Figure 1.A graph depicting the importance of scheduling.

Assignment of priorities is also important when a subgraph at a processor has several interdependent nodes. For example if the whole graph shown in Figure 1 is assigned to a single processor, there are 7 ! possible combinations of execution. Since some of the nodes are interdependent, the processor could waste a lot of time in picking 
up a node for execution and then finding that its predecessor has not been executed yet. If, however, the nodes are first assigned priorities and the processor then executes the nodes on the basis of their priorities, the overhead would be greatly reduced.

Figure 1 further clarifies the difference between our definition of scheduling and that proposed by other researchers. In our scheduling a processor has access to only a subgraph and its nodes for execution. On the contrary, the literature published by other researchers suggests that once partitioned, nodes in a subgraph which are ready for execution can be assigned/scheduled to any of the processors that are free for work.

A scheduler, in our sense, is an algorithm that takes as input a partitioned and allocated task graph and produces as output a priority list of operations in the task graph. An optimal schedule results when the scheduler generates a list of tasks that guarantees the shortest execution time. A scheduling algorithm should execute quickly and its execution time should increase slowly when the task size increases. It makes little sense to use a scheduler that takes a long time to schedule a job that takes only a small time to execute. Granski [13] seconds this opinion by suggesting that incorporating the scheduling mechanism in a computer is an expensive proposition.

Most of the heuristics for list scheduling explained in section 2.3 consider some parameters of the architecture or the task graph itself. These parameters could include the execution time of each node in the task graph, the number of processors in the architecture and the critical path of the node to be scheduled. It is our hypothesis that the ordering of operations at processors can be done without regard to the interprocessor communication. To evaluate this hypothesis we will have to look into some possible ways of mapping a program to processors and then single out those which could show a difference in assigned priorities and execution time under the influence of IPC. To aid further research we define two scheduling methods:

1. Global Schedule : Ordering the whole graph while considering the 
communication (or dependencies) between processors.

2. Local Schedule : Ordering the individual subgraphs allocated to each processor while ignoring any communication arcs between the processors.

Figure 2 shows a difference between the assignment of priorities when global and local scheduling methods are applied to the graph in Figure 1. The numbers inside nodes are their node numbers and those beside the nodes are their respective priorities, with lower numbers indicating higher priorities.

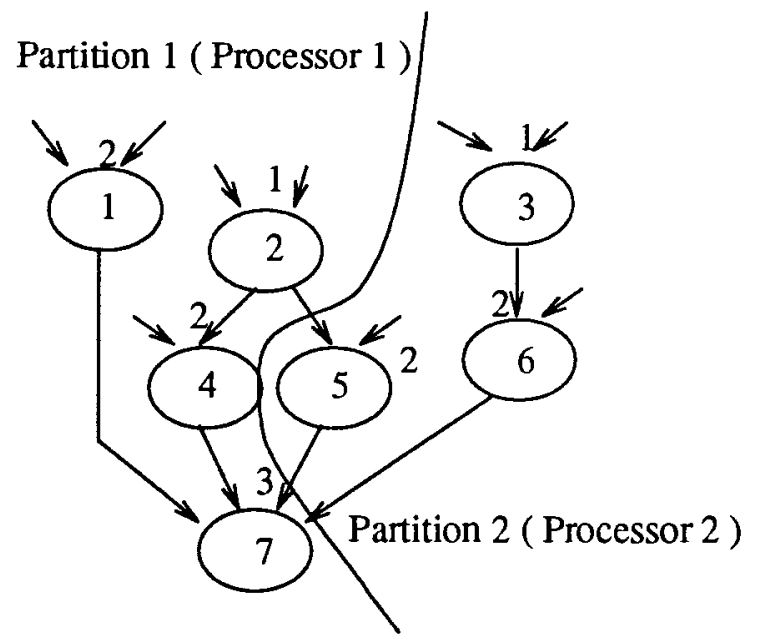

(a) Local schedule

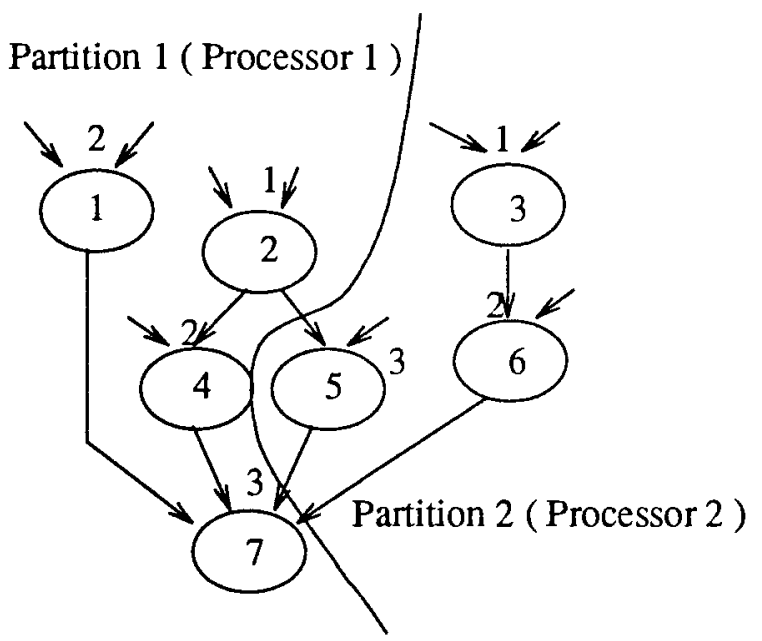

(b) Global schedule

Figure 2.Assignment of Priorities When Graph in Figure 1 is Scheduled Globally and Locally.

It is our hypothesis that ordering of operations at a processor can be done without regard to the interprocessor communication. As seen in Figure 2(a) even after ignoring interprocessor communication there is no change in the assignment of relative priorities to the nodes and thus there will be no change in the execution time either. If put in equation form, we have:

$$
\tau_{g} \approx \tau_{l},
$$




$$
\tau_{g} \approx \tau_{l},
$$

where $\tau_{g}$ and $\tau_{l}$ represent the execution time of globally and locally scheduled graphs.

Also, since the global scheduler considers the interprocessor communication time, its computational complexity will always be greater than or equal to the local scheduler, i.e.,

$$
O(g) \geq O(l)
$$




\section{CHAPTER III}

\section{PARALLEL PROGRAMS AND THEIR INFLUENCE ON GLOBAL SCHEDULES}

\subsection{IMPORTANCE OF PARALLEL PATHS FOR GLOBAL SCHEDULE}

Partitioning, allocating and scheduling tasks for execution on a computer system requires the mapping of the tasks to the computer system hardware in a manner that improves some aspect of system performance. Scheduling thrives on parallelism present in algorithms. A parallel program therefore has to be mapped to an architecture efficiently in order to exploit the parallelism present in it. Consider the parallel program shown in Figure 3.

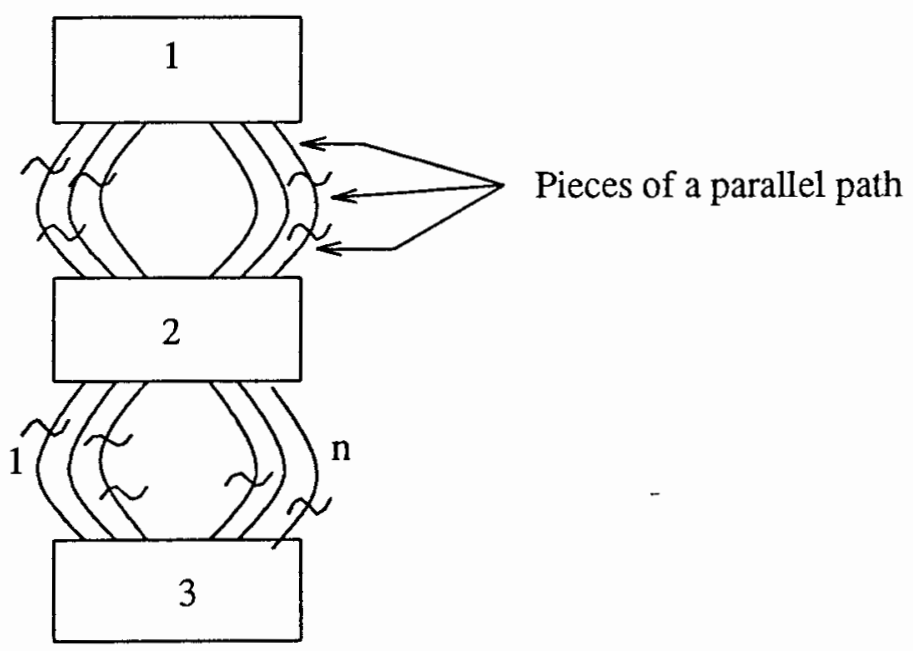

Figure 3.A general parallel program. 
The rectangular blocks represent fork and join points of the program. A fork point, by definition, has two or more independent paths leading out of it and execution must proceed along each and every one of the paths leading out of the fork. Their common output point is called a join point. In Figure 3 blocks 1 and 3 represent fork and join points, respectively, and block 2 represents both. Each block in such a case would represent a barrier [34] in the execution sequence of the program or, in other words, the program would stop and then start its parallel execution at each block. A barrier is a convenient synchronization mechanism among executing processes. The synchronization requires that all processes execute the barrier construct before any process can proceed past it to the next executable statement. Barriers are usually used to satisfy a number of data dependences simultaneously by imposing sequentiality on the production and use of the data items. In the Force language [17], a section of code is inserted between the beginning and end of a barrier construct. This code is executed by one processor after all processes arrive at the barrier and before any process leaves it. Similarly blocks 1 and 3 in Figure 3 could be the startup and ending code, respectively, of a program. This simple model may be extended to a well defined form of concurrent processing which is represented by nodes (instructions) on paths between a pair of fork and join points. Each path in such a case would represent a subtask of the entire program. In fact, barrier synchronization is commonly used in many parallel programming applications [18].

To clarify the analysis further let us define the components of Figure 3. Note that in the discussion that follows we will use path to mean a chain or string of operations in a graph. A path starts at a fork point and ends at a join point. If generalized, the model shown in Figure 3 can be represented as having $n$ parallel paths. During the mapping process the graph would be partitioned and then allocated. During the partitioning phase, depending on the partitioning algorithm being used, the subtasks or paths could be clustered and assigned to the same or different processors and an individual path may be cut (split) into pieces. Applying this terminology to the parallel program shown in 
Figure 3, let $m_{i}$ represent the number of pieces of path $i$ and $P_{k}{ }^{i}, j$ represent the $j$ 'th piece of path $i$ assigned to processor $P_{k}$, where

$$
\begin{aligned}
& 1 \leq i \leq n \\
& 1 \leq j \leq m_{i} .
\end{aligned}
$$

Figure 4 shows a parallel program annotated using this terminology.

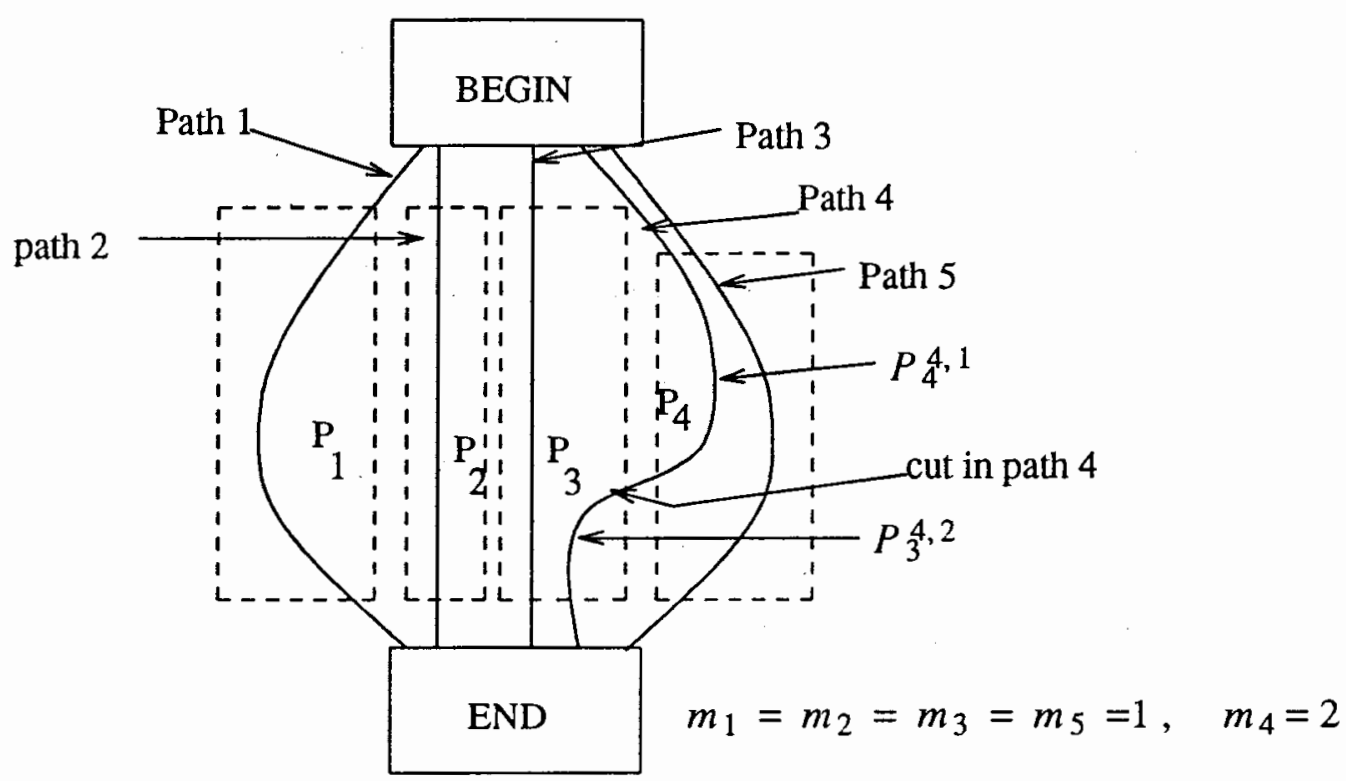

Figure 4.A parallel program with paths assigned to processors.

The multiprocessor architecture model to which parallel programs are mapped consists of a number of homogeneous or heterogeneous processors. The processors communicate via an interconnection network and the amount of time taken to send a fixed amount of data from one processor to another is constant. Each processor in the architecture contains only one arithmetic-logic unit. Computations at processors and communication between them may proceed in parallel subject to the dependency constraints of the algorithm. 


\subsection{SUITABLE SCHEDULING ALGORITHMS}

As described in Chapter II we found that the list scheduling heuristic suggested by Granski [13] is appropriate to finding a suitable model depicting a difference in global and local schedules. Granski's heuristic is based on the critical path algorithm and incorporates a development by which graphs with conditional nodes and loops can be scheduled. The latter case, however, is possible only when the number of iterations of a loop in the graph is known prior to execution. In such cases the loop can be unrolled to produce an acyclic graph, which is then scheduled. The heuristic starts by first assigning weights to all of the strings of nodes in the paths of the graph, where the weight of a string is the sum of the execution times of all nodes in it and the maximum weight of its successor strings. In order to assign weights the algorithm starts with each string $P^{i, m_{i}}$ connected to the join point of a program, assigns it a weight and then assigns weights to strings whose successor strings have already been assigned weights. So if $P^{i, k}$ is a string which is a successor of string $P^{i, j}$ and $T_{m}^{i, j}$ is the time to execute $P^{i, j}$ on processor $m$, then the weight of $P^{i, j}$ can be written as:

$$
W^{i, j}=T_{m}^{i, j}+\max _{P^{i, k} \in \operatorname{succ}}\left(W^{i, k}\right)
$$

where $W^{i, j}$ is the weight of string $P^{i, j}$

While mapping a graph to a multiprocessor system the graph may be partitioned and hence a string in a subgraph could have a predecessor string in another subgraph. In such cases we have to consider the communication time between strings and the equation for the weight of a string would then be:

$$
W^{i, j}=T_{m}^{i, j}+P^{i, k} \in \max \left(W^{i, k}+C^{i, k}\right)
$$

where $C^{i, k}$ is the communication time between strings $P^{i, j}$ and $P^{i, k}$.

Once all strings have been assigned weights, they are assigned priorities in decreasing order of their weights. In situations where two or more strings have the same 
weights, a string having the longest path length from it to the exit node is assigned the highest priority. If Granski's algorithm is applied to the program in Figure 4 and we assume communication time is greater than the execution time of nodes, the pieces above the cut in path 4 will be assigned higher priority than any other path. If $T^{k}$ and $T^{k, j}$ represent the execution time of path $k$ and $j$ 'th piece of path $k$ respectively, the execution sequence generated for Figure 4 would be as shown in Figure 5.

Note that in all of the examples considered hereafter, we will treat the basic operations as a node in a path, i.e., each piece of the path is a primitive operation (node). We use nodes because the algorithm requires deterministic execution times, which are only available for primitive operations.

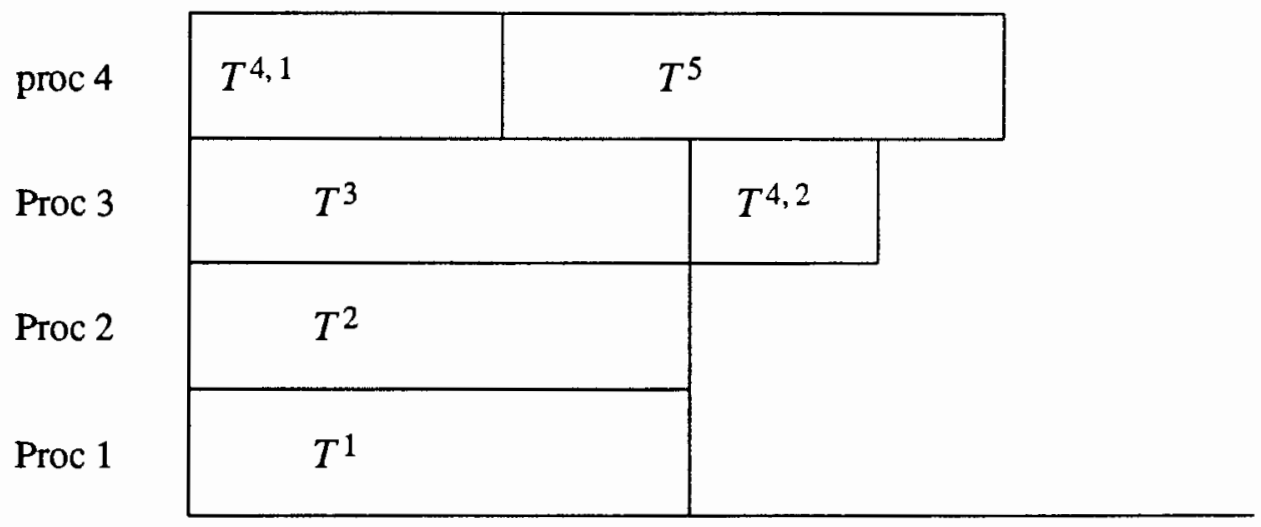

Figure 5.Firing Sequence of Paths in Figure 4 When Scheduled by Granski's Heuristic.

\subsection{IMPORTANCE OF A CUT IN A PATH}

Our hypothesis states that the interprocessor communication does not play an important role in scheduling a set of nodes at a processor. To identify cases where it may make a difference for our program model, we prove the following theorem. 
Theorem

Assignment of relative priorities changes only if a path in a program is cut.

To prove this theorem we have to investigate all possible mappings of a program to an architecture and look for any change in the assignment of priorities and execution time because of interprocessor communication. Note that if relative priorities remain unchanged, execution time will also remain unchanged.

Proof

We analyze two cases of mappings, one in which none of the paths are cut and the second in which one of the paths is cut. We will then study the assignment of priorities to the strings of nodes in each allocation in the global and local schedule cases. Note that path $P \dot{k}, j$ has higher priority than $P k^{,}, m$ if and only if $W^{i, j}-W^{l, m} \geq 0$, where $W^{i, j}$ is the weight of the $j$ 'th piece of path $i$.

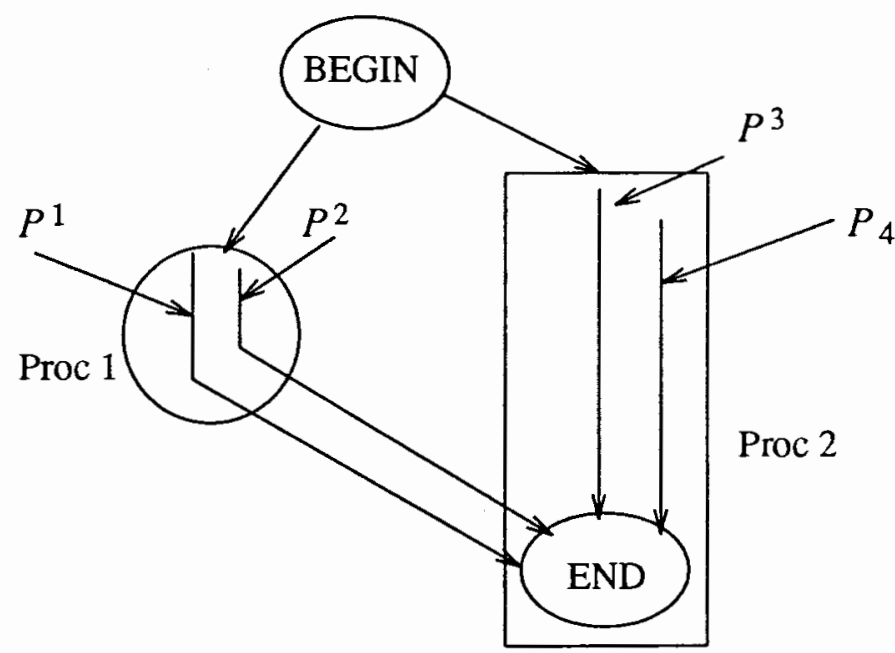

Figure 6.Graph with uncut parallel strings allocated to two processors.

Case 1.a) Path is uncut (End at the different processors). Figure 6 shows a graph whose parallel strings are allocated to two processors and each path terminates at an end 
node residing in processor 2. First consider two arbitrary paths $P^{1}$ and $P^{2}$ at processor 1. Using the notation shown in section 3.2 and global schedule, piece $\mathbf{i}$ of path $P^{1}$ has weight $W_{g}^{1, i}=\sum_{k=i}^{m_{1}} T^{1, k}+C$, i.e, the sum of execution times of the successor paths plus the communication to the end node. Similarly the weight of piece $j$ of path $P^{2}$ for global schedule will be $W_{g}^{2, j}=\sum_{k=j}^{m_{2}} T^{2, k}+C$.

The priority of piece $P^{1, i}$ is higher than $P^{2, j}$ if and only if $W_{g}^{1, i}-W_{g}^{2, j} \geq 0$. If we substitute the weights of path $P^{1}$ and $P^{2}$ in above condition, we get:

$$
\begin{aligned}
& \sum_{k=i}^{m_{1}} T^{1, k}+C-\sum_{k=j}^{m_{2}} T^{2, k}-C \geq 0 \\
& \text { or } \\
& \sum_{k=i}^{m_{1}} T^{1, k}-\sum_{k=j}^{m_{2}} T^{2, k} \geq 0 .
\end{aligned}
$$

The local scheduler does not consider the communication time, so the piece $i$ of path $P^{1}$ and piece $j$ of path $P^{2}$ will have weights as follows.

$$
\begin{aligned}
& W_{l}^{1, i}=\sum_{k=i}^{m_{1}} T^{1, k} \\
& W_{l}{ }^{2, j}=\sum_{k=j}^{m_{2}} T^{2, k}
\end{aligned}
$$

Since there is no relative difference in the local weights of $P^{1, i}$ and $P^{2, j}$ and since the amount of communication between paths $P^{1}$ and $P^{2}$ with the end node is the same, we can therefore conclude that $W_{g}^{1, i}-W_{g}^{2, j} \geq 0$ if and only if $W_{l}^{1, i}-W_{l}^{2, j} \geq 0$, and the relative priorities under global and local schedules are identical.

Case 1.b) Path is uncut (End at the same processors). In Figure 6 paths $P^{3}$ and $P^{4}$ and the end node have been assigned to the same processor. The global weights of paths $P^{1}$ and $P^{2}$ do not change from the global to the local schedule because there is no com- 
munication with the end node and so neither has a higher priority than the other.

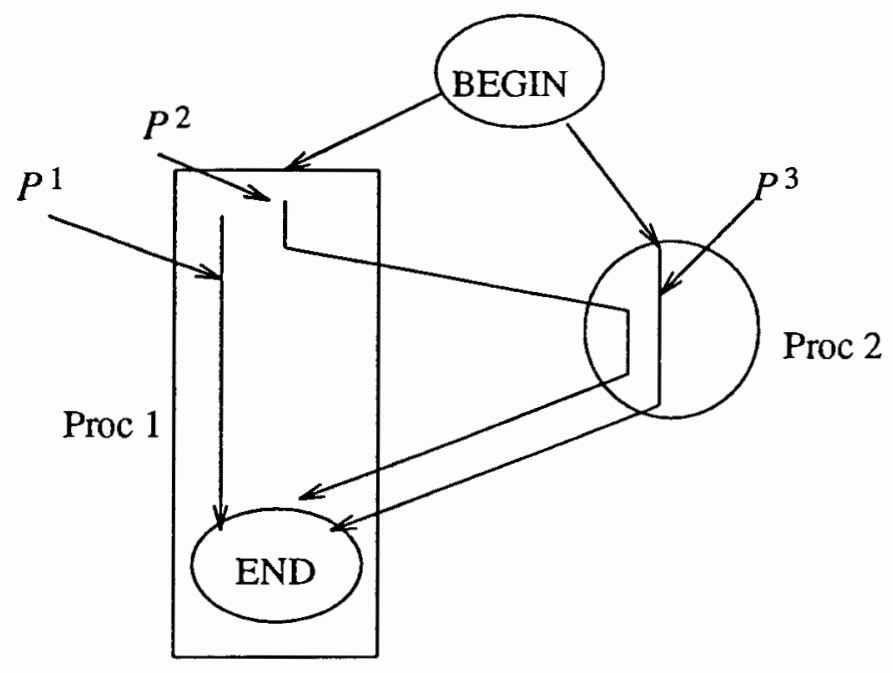

Figure 7.Mapping pieces of a cut path to different processors.

Case 2.a) Path is cut. We will analyze a case in which pieces of a cut path will be assigned to different processors. Figure 7 shows such a mapping. On the basis of the notation developed in section 3.1, the equations for the weights of paths $P^{1}$ and $P^{2}$ under global scheduling can be written as:

For piece i of uncut path $P^{1}: W_{g}^{1, i}=\sum_{k=i}^{m_{1}} T^{1, k}$

For piece j of cut path $2: W_{g}^{2, j}=\sum_{k=j}^{m_{2}} T^{2, k}+n C$

where $n$ is the number of cuts in path 2 and piece $j$ is above the cut.

In the case of the local schedule the weight of piece i of path $P^{1}$ will be the same as global weight but the weight of piece $\mathrm{j}$ of path $P^{2}$ will be

$$
W_{l}^{2, j}=\sum_{k=j}^{m_{i}^{\prime}} T^{2, k}
$$

where $m_{2}^{\prime}<m_{2}$ and is the number of nodes above the cut. In the global schedule case, the relative priority of piece $i$ of path 1 will higher than that of piece $j$ of path 2 if and 
only if $W_{g}^{1, i}-W_{g}^{2, j} \geq 0$

Substituting the global weights for piece $i$ and $j$ we get:

$$
\sum_{k=i}^{m_{1}} T^{1, k}-\sum_{k=j}^{m_{2}} T^{2, k}-n C \geq 0
$$

Now consider the case when $\sum_{k=i}^{m_{1}} T^{1, k}=\sum_{k=j}^{m_{2}^{\prime}} T^{2, k}$. The equation for global weights will then be:

$$
\sum_{k=j}^{m_{j}^{\prime}} T^{2, k}-\sum_{k=j}^{m_{2}} T^{2, k}-n C \geq 0
$$

or

$$
\sum_{k=j}^{m_{2}^{\prime}} T^{2, k} \geq \sum_{k=j}^{m_{2}} T^{2, k}+n C
$$

This case is true only if $n C<0$. Communication time, however, is always greater than 0 and so $\sum_{k=j}^{m_{2}} T^{2, k}+n C$ is always greater than $\sum_{k=j}^{m_{2}} T^{2, k}$. In other words piece $\mathrm{j}$ of path $P^{2}$ will have higher priority than piece i of path $P^{1}$. In the local schedule case, the relative priority of piece $i$ of path 1 will higher than that of piece $j$ of path 2 if and only if $W_{l}^{1, i}-W_{l}^{2, j} \geq 0$

Substituting the local weights for piece $i$ and $j$ we get:

$$
\sum_{k=i}^{m_{1}} T^{1, k}-\sum_{k=j}^{m_{2}^{\prime}} T^{2, k} \geq 0
$$

In such a case when the lengths of piece $i$ of path 1 and piece $j$ of path 2 are same, their weights will be same. In other words, in the local schedule case, piece $i$ of path 1 will have higher priority than piece $j$ of path 2 which is different than the global schedule. We can therefore conclude that the relative priorities in global and local schedule cases are different when a path is cut, proving the theorem. 


\subsection{EXPERIMENTAL FOCUS}

As seen from the above explanations, a major difference in global and local schedule execution times occurs only when a path is cut into multiple pieces. To show our hypothesis, we must now look at the importance of the difference when a path is cut and its effect on the execution time. This analysis will be based on a simple dataflow graph model to be shown in Chapter IV. This model, though being a very specific mapping case, has two parallel paths, one of which is cut into two pieces. Since a cut in a path is all that makes a difference in execution time, the model should be sufficient in designing our experiments. Communication time between processors is a major factor which brings out a difference in execution time when a path is cut. We will vary the number of nodes that reside on the pieces of paths and observe the effect of their overlap with the communication time on the execution of globally and locally scheduled graphs. 


\section{CHAPTER IV}

\section{MODELING SCHEDULE EXECUTION TIMES}

\subsection{AN IDEAL GRAPH}

As previously explained in Chapter III a cut in a path, could result in a significant difference in execution times for global and local scheduling techniques. As a simplification of the graph shown in Chapter III, a simple model graph shown in Figure 8 has been chosen for experiments and in this chapter we have tried to investigate some of its properties.

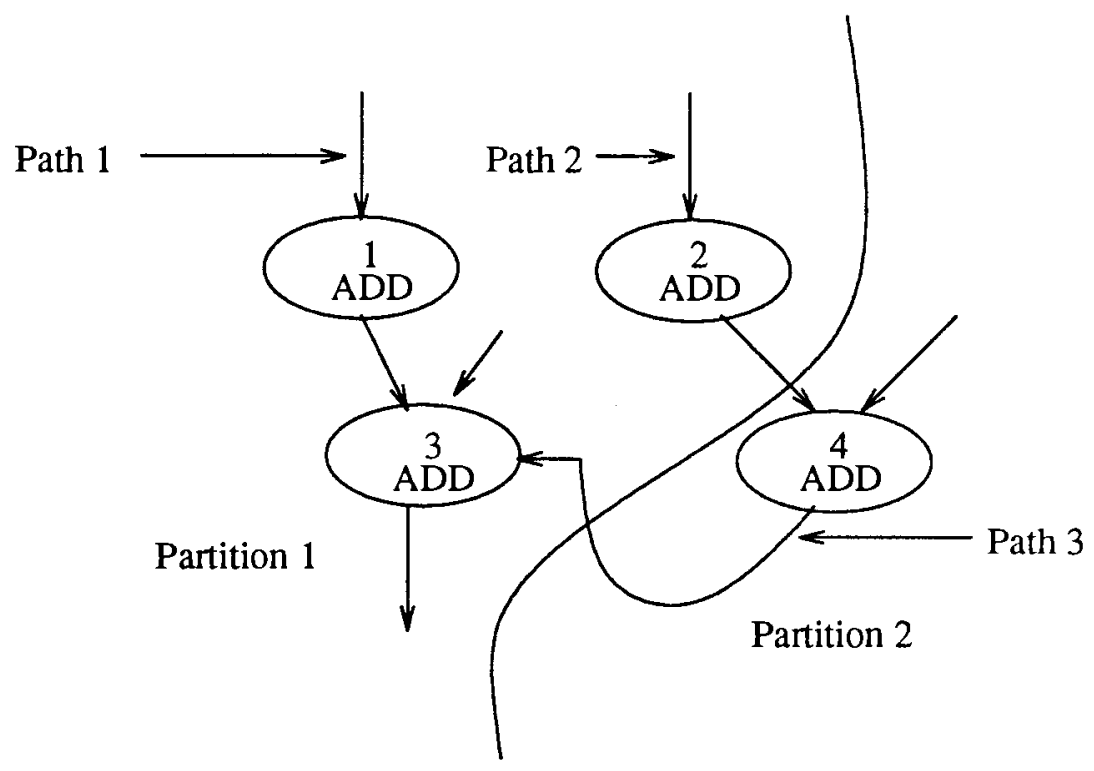

Figure 8.An Ideal Graph for Modeling Execution time of Global and Local Schedules. 
The dataflow graph shown in Figure 8 can be modeled as a set of paths between a fork and a join node as shown in Figure 9.

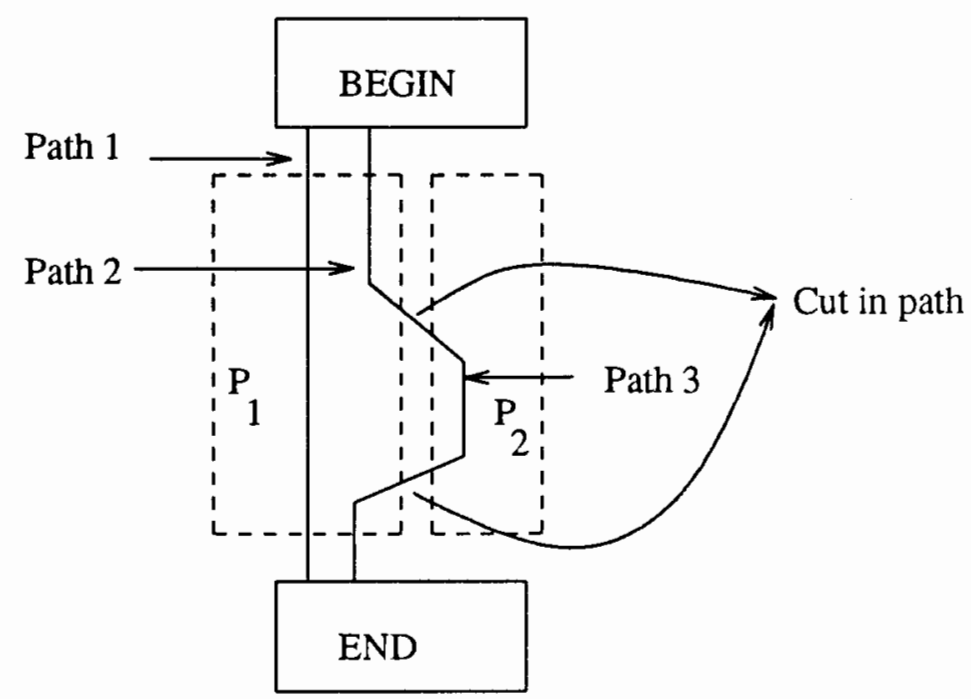

Figure 9.Dataflow Graph in Figure 8 Modeled as a Set of Paths between Fork and Join Points.

We have seen in Chapter III that a difference in execution time of globally and locally scheduled graphs is caused by the overlapping of nodes and the communication time between processors. Since the graph in Figure 8 has three pieces, an analysis of the behavior of this graph is a three dimensional problem.

\subsection{MODELING APPROACH}

Each instruction or node in a program has a finite execution time. For ease in modeling execution times, let us assume that each node in the graph of Figure 8 takes $t$ time units to execute. Similarly assume that the communication time between the partitions is $c$ time units and $c=z \times t$, where $z$ is a positive real number. Note that as assumed in the description of the multiprocessor architecture in section 3.2 , the communication time will be constant irrespective of the kind of instruction (node) being executed. 
communication time will be constant irrespective of the kind of instruction (node) being executed. Assuming that $\mathrm{t}=\mathrm{c}=0.1$, Table I shows the global and local schedules generated for the graph in Figure 8. Before we start modeling the execution times of the globally and locally scheduled graphs let us define some notations which we will use frequently.

$t^{i, j, k}$ represents an overlap between the execution of nodes $\mathrm{i}, \mathrm{j}$ and $\mathrm{k}$ where $1 \leq i, j, k \leq n$ and $n$ is the total number of nodes in the graph.

$c_{l, j}^{k}$ represents an overlap of the communication between nodes $i$ and $j$ and the execution of node $\mathrm{k}$.

TABLE I

SCHEDULE FOR GRAPH IN FIGURE 8

\begin{tabular}{|c|c|c|c|c|}
\hline \multirow{2}{*}{ Actor\# } & \multicolumn{2}{|c|}{ global } & \multicolumn{2}{c|}{ local } \\
\cline { 2 - 5 } & weight & priority & weight & priority \\
\hline 1 & .2 & 3 & .2 & 1 \\
\hline 2 & .5 & 1 & .1 & 2 \\
\hline 3 & .1 & 4 & .1 & 2 \\
\hline 4 & .3 & 2 & .1 & 2 \\
\hline
\end{tabular}

The equations for execution time of the globally and locally scheduled graph can be derived from Table I. Using the notation described above and assuming $c=t$, the equations are:

1. For global schedule:

$$
\tau_{g}=t^{2}+c \frac{1}{2,4}+t^{4}+c_{4,3}+t^{3}=3 t+2 c
$$

2. For local schedule:

$$
\tau_{l}=t^{1}+t^{2}+c_{2,4}+t^{4}+c_{4,3}+t^{3}=4 t+2 c
$$

Figures 10 and 11 show gantt charts which are the execution sequence of nodes in Figure 
8.

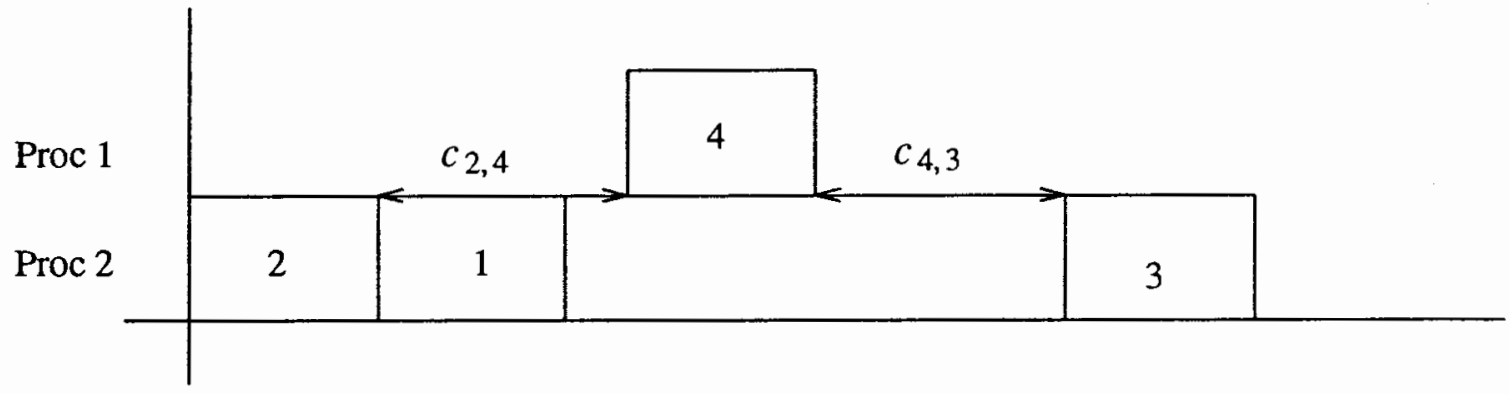

Figure 10.Execution sequence of nodes in Figure 8 for Global schedule.

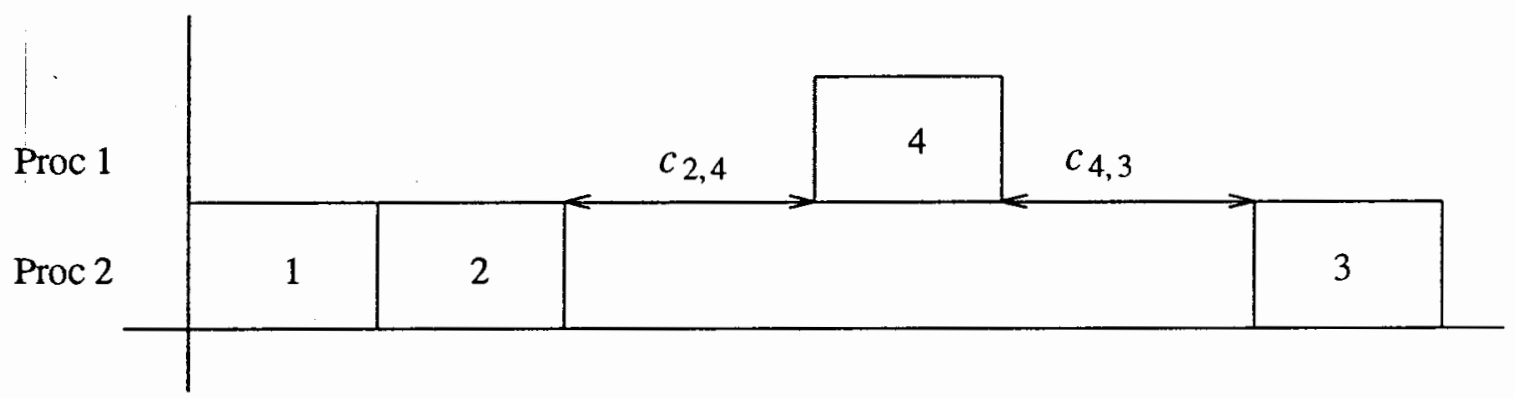

Figure 11.Execution sequence of nodes in Figure 8 for Local schedule.

The scheduling algorithm used assigns priorities to the nodes of the graph on the basis of their weights, where the weight of a node is the sum of its execution time, the maximum weight of its successors and its communication time, if any, with its successors. Since in the case of a global schedule we do consider the communication time between partitions, the nodes in path 2 are assigned higher priority than the nodes in path 1. This results in an earlier firing of nodes in path 2 than those in path 1 and the execution time of the nodes in path 1 are overlapped by the communication time between nodes 2 and 4 .

In the case of the local schedule, since we do not consider the communication 
between the partitions, the nodes in path 1 and path 2 are assigned priorities in an alternating fashion. This results in node 1 firing before node 2 and no overlap of nodes occurs with the communication between node 2 and node 4 . As seen from the above interpretation the key parameters which bring out different schedules and different execution times are:

1. The communication time between the allocations.

2. Length of paths 1,2 and 3.

3. The execution time of the nodes in the graph.

4. Scheduling algorithms used.

If this is put in an equation form, we have:

$$
\left.T=\tau_{l}-\tau_{g}=f \text { (list scheduling }, t, c \text {, path lengths }\right)
$$

Since the graph's behavior depends on the number of nodes in paths 1 and 2 and also on the number of nodes in path 3, an analysis of the behavior will be a three dimensional problem. In the following sections we will analyse the behavior of the graph for varying the number of nodes in paths 1,2 and 3 .

\subsection{GRAPH BEHAVIOR VERSUS VARIATIONS IN KEY PARAMETERS}

To ease an understanding of the analysis that will follow we will first put forward some naming conventions:

1. $n_{p^{1}}=$ the number of nodes in path 1 .

2. $n_{p^{2}}=$ the number of nodes in path 2 .

3. $n_{p^{3}}=$ the number of nodes in path 3 .

4. $r=$ the number of arcs between the two partitions.

As suggested in section 4.2 the difference in execution time of globally and locally scheduled graphs is a function of the lengths of paths 1,2 and 3. To predict the 


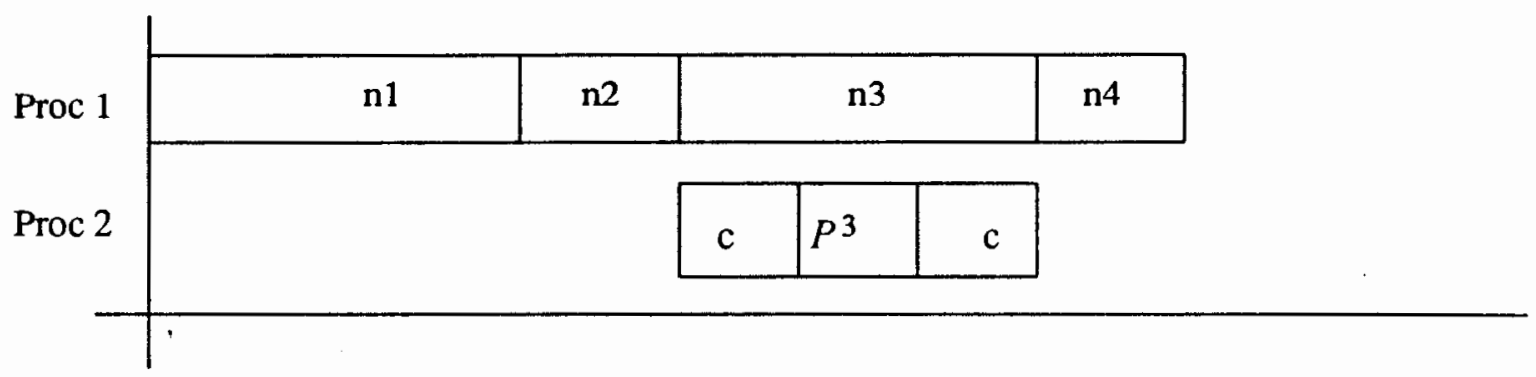

Figure 12.General Execution Sequence for Globally Scheduled Graph in Figure 8.

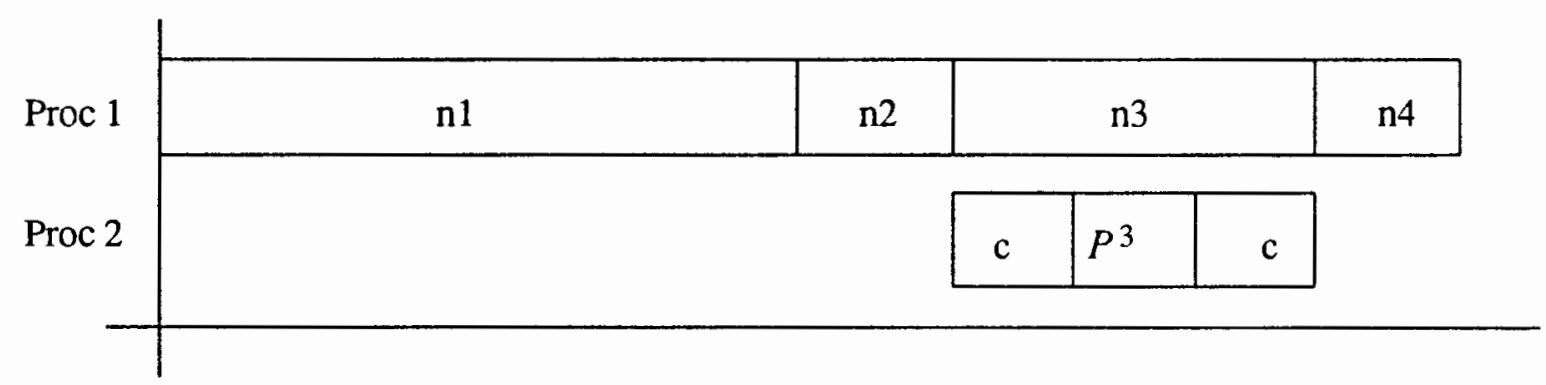

Figure 13.General execution sequence for Locally scheduled graph in Figure 8.

\subsubsection{Global schedule}

Figure 12 shows the general execution sequence of the graph when scheduled globally. Let us analyse each stage of execution.

Stage 1. Depending on the length of paths 1,2 and 3 some of the nodes in path 1 or path 2 may execute alone or may execute alternately. Let $n 1$ be the number of nodes of either path 1 or path 2 that execute alone with no overlap. If $n 1 a$ and $n 1 b$ are the number of nodes of path 1 and path 2 respectively which execute alone, then n1a will be:

$$
\mathrm{n} 1 \mathrm{a}=\max \left(0, n_{p^{1}}-n_{p^{2}}-n_{p^{3}}-\frac{r c}{t}\right)
$$

where $\left(n_{P^{1}}-n_{p^{2}}-n_{p^{3}}-\frac{r c}{t}\right)$ is the number of nodes in path $P^{1}$ with higher priority than the nodes in path $P^{2}$.

Similarly n1b will be: 
where $\left(n_{P^{1}}-n_{p^{2}}-n_{p^{3}}-\frac{r c}{t}\right)$ is the number of nodes in path $P^{1}$ with higher priority than the nodes in path $P^{2}$.

Similarly $\mathrm{n} 1 \mathrm{~b}$ will be:

$$
\mathrm{n} 1 \mathrm{~b}=\max \left(0, \min \left(n_{p^{2}}+\frac{r c}{t}+n_{p^{3}}-n_{p^{1}}, n_{p^{2}}\right)\right)
$$

where $n_{p^{2}}+\frac{r c}{t}+n_{p^{3}}-n_{p^{1}}, n_{p^{2}}$ is the number of nodes in path $P^{2}$ with higher priority than $P^{1}$ if path $P^{1}$ is longer than $P^{2}$. Also note that $\mathrm{n} 1=\mathrm{n} 1 \mathrm{a}+\mathrm{n} 1 \mathrm{~b}$.

Stage 2. Let $n_{p^{1}}^{\prime}$ be the number of nodes left in path 1 after executing nla nodes and $n_{p^{2}}^{\prime}$ be the number of nodes left in path 2 after executing $n 1 b$ nodes. The equations for $n_{p^{1}}^{\prime}$ and $n_{p^{2}}^{\prime}$ can be written as:

$$
\begin{aligned}
& n_{p^{1}}^{\prime}=n_{p^{1}}-n 1 a, \\
& n_{p^{2}}^{\prime}=n_{p^{2}}=n 1 b .
\end{aligned}
$$

These nodes left in paths 1 and 2 may fire alternately depending on their numbers. When $n_{p^{1}}^{\prime}$ is larger than $n_{p^{2}}^{\prime}$, then $n_{p^{2}}^{\prime}$ will be exhausted after $2 n_{P^{2}}^{\prime}$ since one node is executing from each path. In the case when $n_{p^{2}}^{\prime}$ is larger than $n_{p^{1}}^{\prime}$, we exhaust $n_{p^{1}}^{\prime}$ early and then continue with $n_{p^{2}}^{\prime}$. In Figure $12, \mathrm{n} 2$ is the number of nodes firing alternately in paths 1 and 2. The equation for $\mathrm{n} 2$ can then be written as:

$$
\mathrm{n} 2=\min \left(2 \times n_{p^{2}}^{\prime}, n_{p^{2}}^{\prime}+n_{p^{1}}^{\prime}\right)
$$

Stage 3. At this point the nodes in path 2 are completely exhausted and some nodes in path 1 may remain unexecuted. In Figure 12, n3 is the number of nodes in path 1 whose execution could overlap with the communication arcs and the nodes in path 3 , or in equation form:

$$
\mathrm{n} 3=\frac{r c}{t}+n_{p^{3}}
$$

Stage 4. After executing $\mathrm{n} 3$ nodes from path 1 some nodes may still be remaining 
12 is the number of nodes executed at stage 4 , hence

$$
\mathrm{n} 4=n_{p}^{\prime \prime}
$$

\section{$\underline{4.3 .2 \text { Local schedule }}$}

Figure 13 shows the execution sequence when the graph is scheduled locally.

Stage 1. Since the local scheduler does not consider the communication time when assigning weights to nodes of path 1 and path 2, more nodes in path 1 and path 2 will execute alone. $\mathrm{n} 1 \mathrm{a}$ and $\mathrm{n} 1 \mathrm{~b}$ in stage 1 of the local schedule case will be:

$$
\begin{aligned}
& \mathrm{n} 1 \mathrm{a}=\max \left(0, n_{p^{1}}-n_{p^{2}}\right) \\
& \mathrm{n} 1 \mathrm{~b}=\max \left(0, n_{p^{2}}-n_{p^{1}}\right)
\end{aligned}
$$

Stage 2. The number of nodes left in path 1 and path 2 after executing $n 1 a$ and $\mathrm{n} 1 \mathrm{~b}$ nodes from path 1 and 2 will remain the same as those in global schedule.

$$
\begin{aligned}
& n_{p^{1}}^{\prime}=n_{p^{1}}-n 1 a \\
& n_{p^{2}}^{\prime}=n_{p^{2}}=n 1 b \\
& \mathrm{n} 2=\min \left(2 \times n_{p^{2}}^{\prime}, n_{p^{2}}^{\prime}+n_{p^{1}}^{\prime}\right)
\end{aligned}
$$

Stage 3. The nodes left in path 1 can be overlapped by the communication between processors and the nodes in path 3 .

$$
\mathrm{n} 3=\frac{r c}{t}+n_{p^{3}}
$$

Stage 4. The equations for nodes left in path 1 for execution after stage 3 will be the same as in global schedule case.

$$
n_{p^{1}}^{\prime \prime}=\min \left(n_{p^{1}}^{\prime}-\frac{n 2}{2}-n 3,0\right)=\mathrm{n} 4
$$

Let us look at four examples in which we will try to predict the execution time of the graph towards variation in lengths of paths 1,2 and 3. The three examples are: 
the same as in global schedule case.

$$
n_{p^{1}}^{\prime \prime}=\min \left(n_{p^{1}}^{\prime}-\frac{n 2}{2}-n 3,0\right)=\mathrm{n} 4
$$

Let us look at four examples in which we will try to predict the execution time of the graph towards variation in lengths of paths 1,2 and 3. The three examples are:

1. Lengths of paths 1 and 2 are the same.

2. Length of path 1 is greater than path 2 .

3. Length of path 2 is greater than path 1 .

Lengths of paths 1 and 2 are the same

If we increase the lengths of path 1 and path 2 , a linear increase in the difference of execution time of global and locally scheduled graphs is expected to a certain point, after which it should remain constant. The above statement is based on the following interpretation:

1. Assuming that the communication time is greater than the execution time, i.e., $c=z * t$, the number of nodes in path 1 covered by the communication time would depend on how big " $z$ " is. In case of globally scheduled graphs, as we increase the number of input nodes to path 1 and path 2, the number of overlapping nodes in path 1 approaches a maximum value. The limit on the maximum number of overlapping nodes in path 1 can be given by the following simple equation:

$$
\text { maximum number of overlapping nodes }=\min \left(n_{P^{1}}, \frac{n_{P^{3}} \times t+r \times c}{t}\right)
$$

where $\left(n_{P^{3}} \times t+r \times c\right)$ is the time available for overlap and $t$ is the execution time of a node. Once crossed this number remains the same no matter how many input nodes are added to path 1 and path 2 .

2. On further increasing the number of input nodes to paths 1 and 2, the weights of 
nodes on path 1 of partition 1 start matching those on path 2 . As a result the nodes in path 1 and path 2 of partition 1 will be fired in alteration. This resembles to execution of stage 2 in Figure 12.

In the case of locally scheduled graphs, since the nodes in path 1 and path 2 are fired in succession alternately, no nodes are covered by the communication arcs between the partitions and by node 4 irrespective of the number of nodes in path 1 and path 2. The execution time of the locally scheduled graph therefore increases linearly in $n_{P^{2}}$ and $n_{P^{1}}$.

The equations for the execution time of globally and locally scheduled graphs would be as follows.

1. For global schedule:

$$
\tau_{g}=\left(n_{P^{1}}+n_{P^{2}}+n_{P^{3}}-n 3\right) t+r c \quad \text { if } 0<t<c
$$

2. For local schedule:

$$
\tau_{l}=\left(n_{P^{1}}+n_{P^{2}}+n_{P^{3}}\right) t+r c
$$

In such a case when the execution time of nodes in the graph is more than the communication time between partitions i.e, $\mathrm{c}=\mathrm{z} \mathrm{t}, 0<\mathrm{z}<1$, only a few or none of the nodes in path 1 of partition 1 would overlap the communication between the partitions. This would, however, depend on how small " $z$ " is. In case $z \rightarrow 0$ or, in other words, the execution time of a node is far larger than the communication time between processors, one can neglect the communication time. In such a case, the equation for global schedule would be the same as for local schedule i.e, $\tau_{g}=\tau_{l}=\left(n_{P^{1}}+n_{P^{2}}+n_{P^{3}}\right) t$.

\section{Length of path 1 is greater than path 2}

On increasing the length of path 1 , a steady increase in the number of nodes covered in path 1 is expected till $n_{p^{1}}=n_{P^{3}}+\frac{r c}{t}$, after which it should remain constant. In case of the local schedule, no nodes would be covered by the communication between 
the partitions, its execution time therefore will increase linearly in $n_{P 1}$. The difference in the execution time of global and local schedules should therefore increase linearly first and then become constant. The equations for the execution time would be:

1. global schedule:

$$
\begin{array}{rr}
\tau_{g}=\left(n_{P^{2}}+n_{P^{3}}\right) t+r c & \text { for } n_{P^{1}} \leq n_{P^{3}} \\
\tau_{g}=\left(n_{P^{1}}+n_{P^{2}}+n_{P^{3}}-n 3\right) t+r c & \text { for } n_{P^{1}} \geq n_{P^{3}}+n 4
\end{array}
$$

where $n 4$ is the number of extra nodes in path 1 that cannot be covered by the communication time and the nodes in path 3.

2. For local schedule:

$$
\tau_{l}=\left(n_{P^{1}}+n_{P^{2}}+n_{P^{3}}\right) t+r c
$$

\section{Length of path 2 is greater than path 1}

The behavior of the graph in this case is similar to that when path 1 is greater than path 2. The equations for the global and local execution are:

1. For global schedule:

$$
\begin{array}{rr}
\tau_{g}=\left(n_{P^{2}}+n_{P^{3}}\right) t+r c & \text { for } n_{P^{1}} \leq n_{P^{3}} \\
\tau_{g}=\left(n_{P^{1}}+n_{P^{2}}+n_{P^{3}}-n 3\right) t+r c & \text { for } n_{P^{1}} \geq n_{P^{3}}+n 4
\end{array}
$$

2. For local schedule:

$$
\tau_{l}=\left(n_{P^{1}}+n_{P^{2}}+n_{P^{3}}\right) t+r c
$$

\subsection{BEHAVIORAL PREDICTION OF EXPERIMENTAL OUTCOME}

As explained earlier, the communication time is a major parameter responsible for the expected linear growth in the difference of execution time of the globally and locally scheduled graphs. The experimentally evaluated values of the communication time between two processors and that of the execution time of an actor of datatype ADD 
in ParPlum [10] are $0.16 \mathrm{sec}$ and 0.014 seconds, approximately. On the basis of these measured values the limit on the number of overlapping nodes in case 4.3 .1 will therefore be:

Maximum number of overlapping nodes $=\frac{0.16 \times 2}{0.014}+1=23.8$

We should therefore expect the difference in execution time of the globally and locally scheduled graphs to increase linearly until the number of input nodes to path 1 reaches the above limit and then become a constant. For 24 input nodes the difference in execution time as calculated by equations 2 and 3 would be :

$$
\tau_{l}-\tau_{g}=1.063-0.729=0.334 \text { seconds. }
$$

As seen from the models in section 4.4, the difference in execution time of globally and locally scheduled graphs is dependent on the size of communication time. Since the communication time is approximately 12 times larger than the execution time, the number of nodes overlapping the communication would not be large. The difference in all of the above cases should therefore not be significant. We ran experiments varying $n_{p^{1}}, n_{p^{2}}$ and $n_{p^{3}}$ to see how the execution time of the graph matches that predicted by the models. The results of the experiments are discussed in Chapter V. 


\section{CHAPTER V}

\section{EXPERIMENTAL RESULTS AND THEIR ANALYSIS}

\subsection{VARIATION IN PATHS 1 AND 2 (PATH 3 CONSTANT)}

Tests were run on Parplum interpreter using a network of Sun workstations for variations in the number of nodes ranging from 1 to 1200 for paths 1 and 2 . The wall clock time, which is also the total execution time in ParPlum, is the sum of the following [10]:

1. Setup time: This is the time to create communication channels among all processes.

2. Parser time: This is the time to parse the graph description and the machine description.

3. Build link time: This is the time to build links between nodes in the graph.

4. Child execution time: This is the time taken by the child processes to execute parts of graph assigned to them.

The graphs were partitioned as shown in Figure 8 and the number of partitions were always kept constant at 2 . Table II shows the data gathered for total execution time for ten test runs. The expected time as predicted by the models in Chapter IV is the child execution time. The expected time as shown in Table II is, however, a sum of the expected setup time, parser time, build link time and the child execution time predicted by our models. While gathering experimental data, the wall clock execution time and the 
child execution time varied by as much as 50 percent due to variations in the load over the network. To get a close match between the actual and expected results, the measured wall clock execution time and the child execution time were averaged over 20 iterations. Figure 14 shows the measured total execution time of the graph using global and local scheduling techniques and Figure 15 shows the difference in execution time of wall clock between the expected and measured results.

\section{TABLE II}

\section{EXECUTION TIME FOR GRAPHS WITH PATH 1 AND 2 VARYING SIMULTANEOUSLY}

\begin{tabular}{|c|c|c|c|c|c|r|}
\hline \multicolumn{4}{|c|}{ Global schedule } & \multicolumn{3}{c|}{ Local schedule } \\
\hline $\begin{array}{c}\text { Number } \\
\text { of nodes }\end{array}$ & $\begin{array}{c}\text { Wall } \\
\text { clock(sec) }\end{array}$ & $\begin{array}{c}\text { Overlapping } \\
\text { nodes }\end{array}$ & $\begin{array}{c}\text { expected } \\
\text { time(sec) }\end{array}$ & $\begin{array}{c}\text { Number } \\
\text { of nodes }\end{array}$ & $\begin{array}{c}\text { Wall } \\
\text { clock(sec) }\end{array}$ & $\begin{array}{c}\text { expected } \\
\text { time(sec) }\end{array}$ \\
\hline \hline 16 & 34.36 & 6 & 36.00 & 16 & 35.36 & 36.00 \\
\hline 26 & 32.36 & 5 & 33.00 & 26 & 34.36 & 36.00 \\
\hline 106 & 66.50 & 14 & 68.00 & 106 & 95.89 & 92.00 \\
\hline 306 & 110.25 & 4 & 109.80 & 306 & 95.34 & 91.00 \\
\hline 406 & 150.10 & 6 & 145.00 & 406 & 190.70 & 170.00 \\
\hline 506 & 177.40 & 6 & 183.70 & 506 & 185.10 & 181.00 \\
\hline 606 & 250.39 & 13 & 254.00 & 606 & 350.19 & 341.00 \\
\hline 706 & 295.12 & 12 & 301.00 & 706 & 285.69 & 288.00 \\
\hline 1006 & 545.59 & 3 & 541.70 & 1006 & 562.62 & 551.90 \\
\hline 1206 & 739.62 & 19 & 727.00 & 1206 & 755.22 & 760.00 \\
\hline 1406 & 1067.00 & 11 & 1074.00 & 1406 & 1207.19 & 1197.00 \\
\hline
\end{tabular}

As seen in Figure 14 the difference in execution time of the globally and locally scheduled graphs was found to be small first and then increasing. The number of nodes overlapping both the communication arcs was, however, found to be slightly erratic and not increasing linearly as expected in section 4.3.1. 


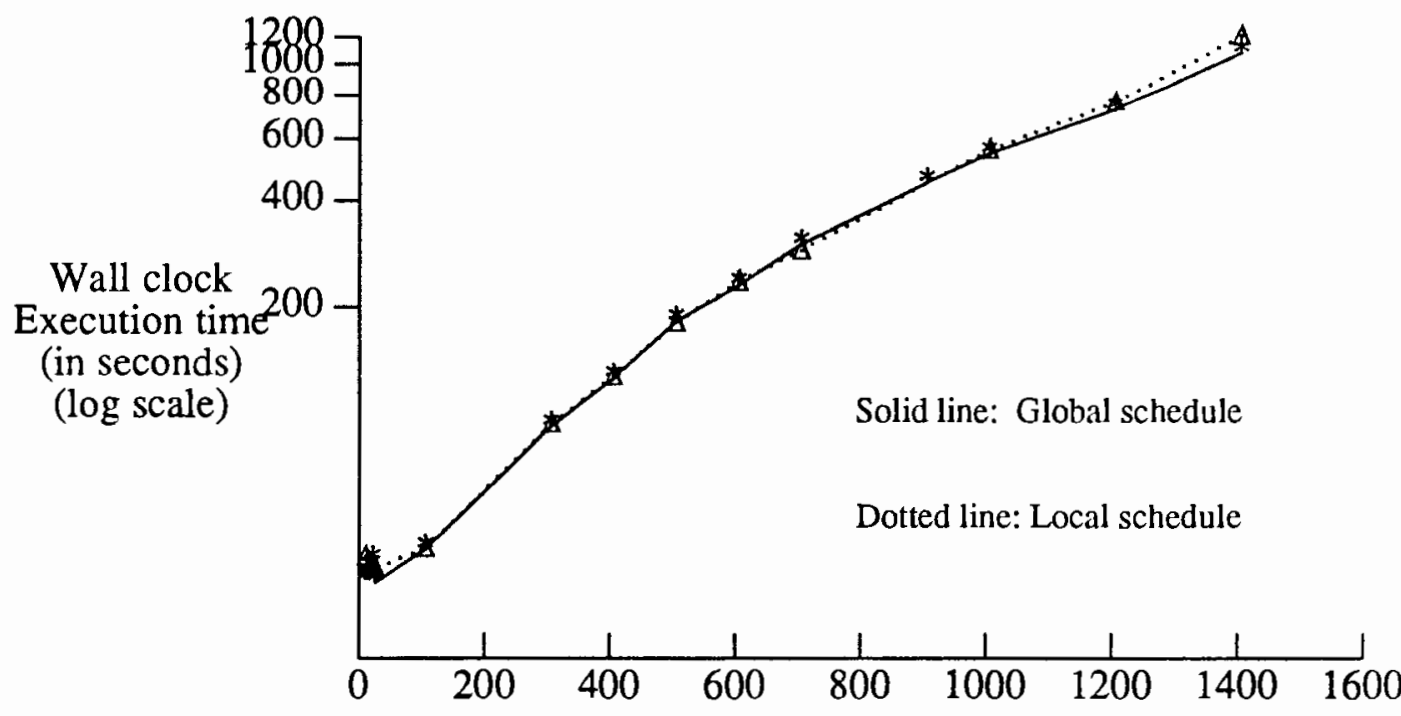

Graph size: Total number of nodes in graph

Figure 14.Graph of Varitation in number of Nodes in Path 1 and 2 Versus total Execution time of Globally and Locally Scheduled Graph.

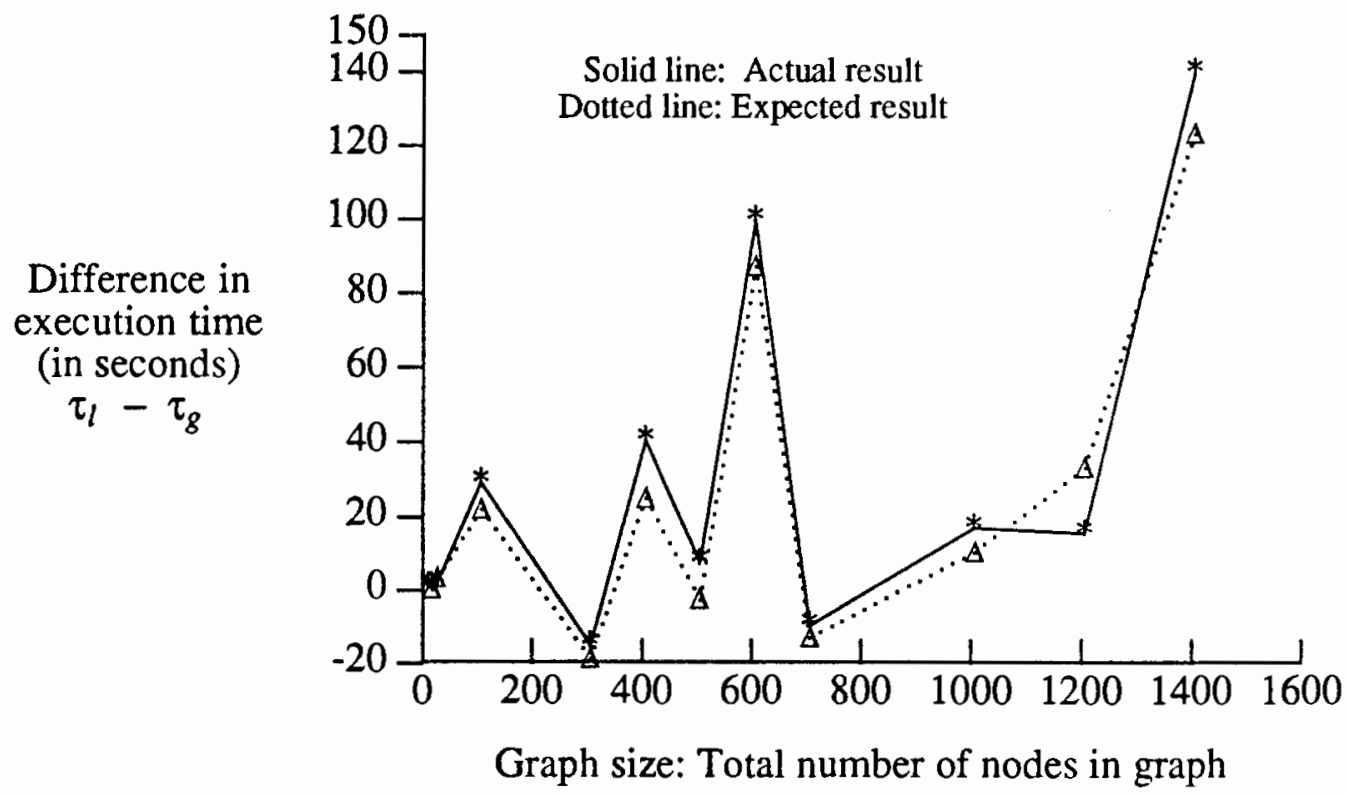

Figure 15.Graph of Varitation in number of Nodes in Path 1 and 2 Versus Difference in total Execution time. 


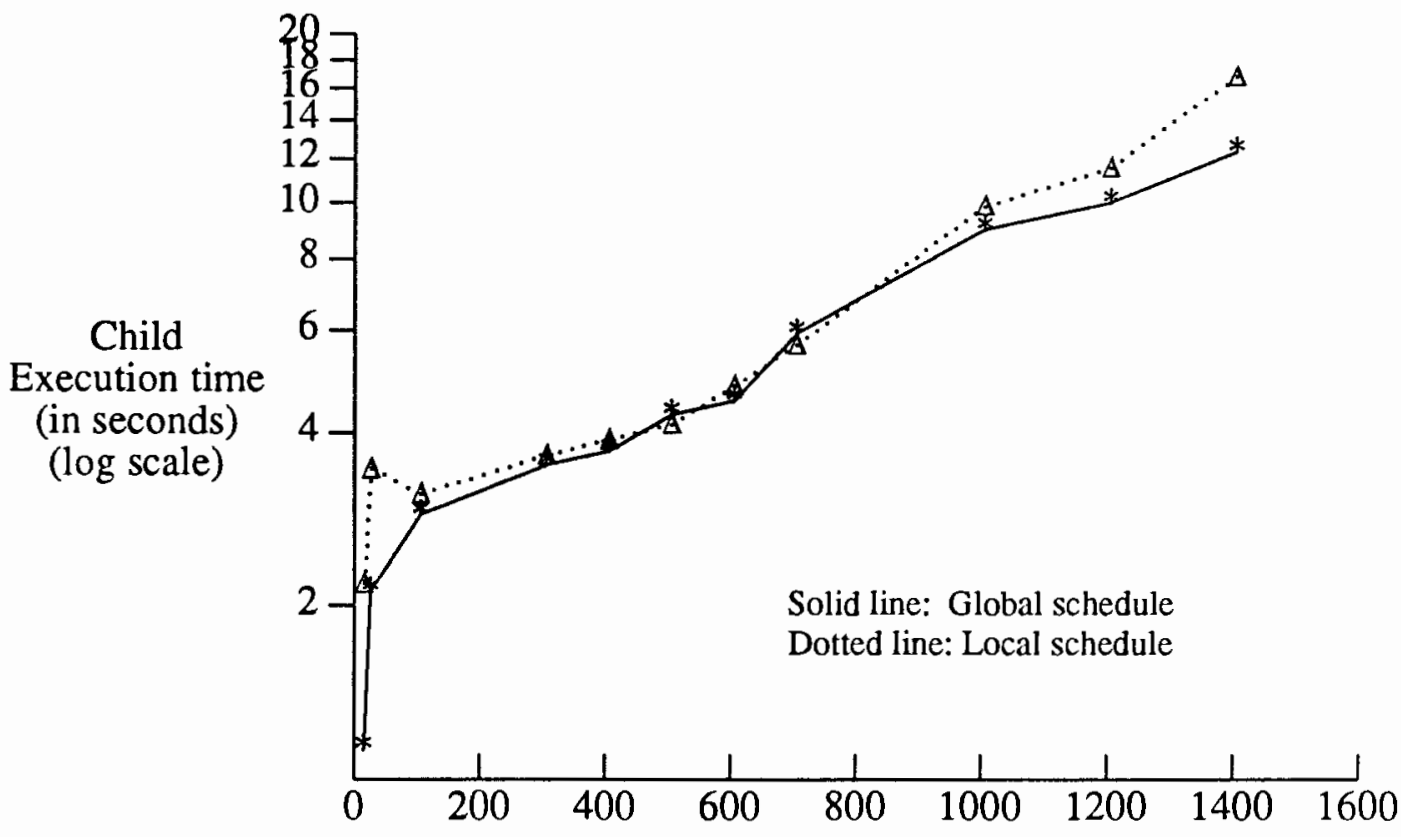

Graph size: Total number of nodes in graph

Figure 16.Child Execution time for Globally and Locally Scheduled Graph in Figure 8.

Since the total execution time (measured) is dominated by the parser time and build link times, they may overshadow the small difference, if any, in the child execution time. Figure 16 therefore shows the measured child execution time in the global and local schedule cases. Since the number of overlapping nodes is governed by two parameters : execution time of a node and the communication time between processors, the number of overlapping nodes and these two parameters were measured again.

1. The execution time of the actors in path 1: The execution time of the nodes in path 1 is found to be 0.014 . Note that this Figure is an average of 100 measurements. Assuming that the actual execution time during runtime varies by $10 \%$ from the estimated value during each simulation, it will still be insufficient to cause the erratic increase in the number of overlapping nodes at times. The reason being that even with a $10 \%$ variation in execution time the number of overlapping nodes will increase or decrease by only $5 \%$ and not by $100 \%$ as seen at times in 
nodes will increase or decrease by only $5 \%$ and not by $100 \%$ as seen at times in Table II.

2. The interprocessor communication time was measured approximately once every half hour over the course of a week [7]. As can be seen from Figure 17, it is not unusual for the time to fluctuate as much as $100 \%$ from its mean value.

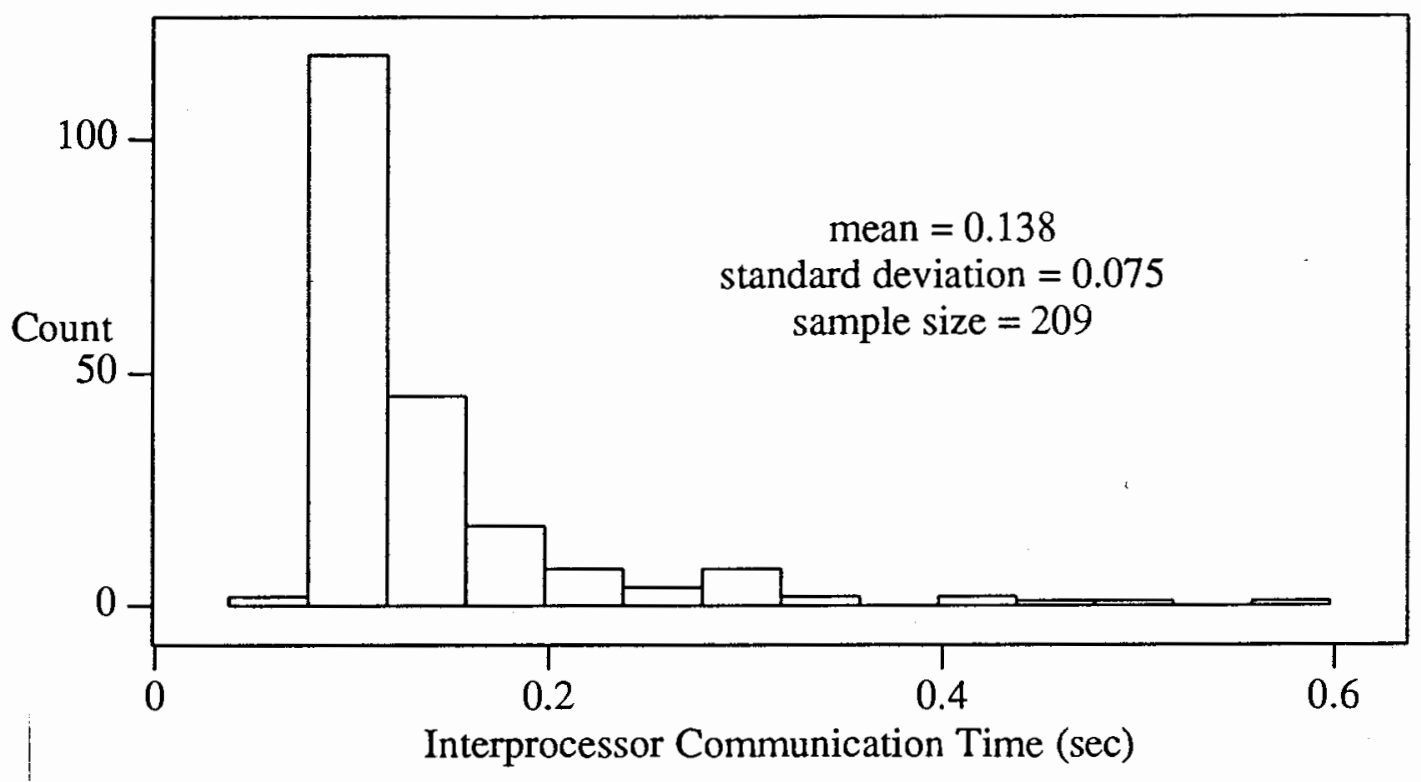

Figure 17. Variation in IPC.

The difference in execution time between expected and actual results can be blamed upon the load average during each simulation.

\subsection{VARIATION IN PATH 3 (PATH 1 AND 2 CONSTANT)}

Tests were run for variations in the number of nodes in path 3 ranging from 1 to 300. These tests were repeated for nodes in path 1 and 2 varying from 50 to 200 . The data generated from those tests is shown in Tables III and IV under the columns with heading 50, 100 and 200 and Figures 18, 19 and 20 show the difference in child 
execution time for the expected and actual results. As seen in the graph, the number of nodes in path 1 overlapping with those in path 3 increases linearly as expected and then becomes a constant once the number of nodes in path 1 equals that in path 3 .

\section{TABLE III}

EXECUTION TIME FOR GRAPHS WITH PATH 3 VARYING (GLOBAL SCHEDULE)

\begin{tabular}{|c|c|c|c|c|c|c|c|c|c|}
\hline \multirow{2}{*}{$\begin{array}{l}\text { Nodes in } \\
\text { path } 3\end{array}$} & \multicolumn{3}{|c|}{$\begin{array}{l}\text { Wall clock time } \\
\text { (measured in sec.) }\end{array}$} & \multicolumn{3}{|c|}{$\begin{array}{c}\text { Overlapping nodes } \\
\text { in path } 1\end{array}$} & \multicolumn{3}{|c|}{$\begin{array}{l}\text { Wall clock time } \\
\text { (expected in sec.) }\end{array}$} \\
\hline & 50 & 100 & 200 & 50 & 100 & 200 & 50 & 100 & 200 \\
\hline 1 & 55.6 & 64.06 & 129.92 & 8 & 9 & 6 & 55.62 & 64.01 & 129.85 \\
\hline 3 & 55.7 & 64.11 & 129.92 & 8 & 10 & 7 & 55.65 & $\overline{64.02}$ & 129.87 \\
\hline 5 & 55.7 & 64.16 & 129.92 & 10 & 13 & 9 & 55.65 & 64.01 & 129.87 \\
\hline 7 & 55.69 & 64.06 & 129.94 & 11 & $\overline{16}$ & 10 & 55.66 & 63.99 & 129.88 \\
\hline 9 & 55.69 & 64.04 & 129.90 & 13 & 19 & 12 & 55.66 & 63.98 & 129.88 \\
\hline 21 & 55.7 & 63.87 & 129.91 & 24 & 40 & 26 & 55.83 & 63.85 & 129.85 \\
\hline 25 & 55.73 & 63.83 & 131.46 & 16 & 47 & 35 & 55.84 & 63.81 & 129.78 \\
\hline 50 & 55.17 & 65.18 & 129.92 & 50 & 50 & 50 & 55.72 & 64.12 & 129.92 \\
\hline 80 & 56.19 & 65.23 & 129.9 & 51 & 76 & 83 & 56.29 & 64.18 & 129.88 \\
\hline 100 & 56.47 & 65.22 & 129.91 & 51 & 97 & 105 & 56.40 & 64.16 & 129.85 \\
\hline 250 & 84.58 & 95.28 & 136.68 & 51 & 101 & 200 & 88.51 & 94.81 & 133.63 \\
\hline 300 & & 95.98 & .138 .38 & & 101 & 200 & & 95.51 & 134.33 \\
\hline
\end{tabular}


TABLE IV

EXECUTION TIME FOR GRAPHS WITH PATH 3 VARYING (LOCAL SCHEDULE)

\begin{tabular}{|c|c|c|c|c|c|c|}
\hline \multirow{2}{*}{$\begin{array}{c}\text { Nodes in } \\
\text { path 3 }\end{array}$} & \multicolumn{3}{|c|}{$\begin{array}{c}\text { Wall clock time } \\
\text { (measured in sec.) }\end{array}$} & \multicolumn{3}{c|}{$\begin{array}{c}\text { Wall clock time } \\
\text { (expected in sec.) }\end{array}$} \\
\cline { 2 - 7 } & 50 & 100 & 200 & 50 & 100 & 200 \\
\hline 1 & 55.80 & 64.2 & 130.01 & 55.73 & 64.13 & 129.85 \\
\hline 3 & 55.83 & 64.23 & 130.04 & 55.37 & 64.16 & 129.97 \\
\hline 5 & 55.86 & 64.26 & 130.06 & 55.79 & 64.19 & 129.99 \\
\hline 7 & 55.89 & 64.29 & 130.09 & 55.82 & 64.22 & 130.02 \\
\hline 9 & 55.91 & 64.32 & 130.13 & 55.84 & 64.25 & 130.05 \\
\hline 21 & 56.08 & 64.48 & 130.29 & 56.01 & 64.41 & 130.22 \\
\hline 25 & 56.08 & 64.54 & 130.29 & 56.07 & 64.47 & 130.28 \\
\hline 50 & 56.49 & 65.89 & 130.7 & 56.42 & 64.82 & 130.63 \\
\hline 80 & 56.91 & 66.31 & 132.27 & 56.84 & 65.84 & 131.05 \\
\hline 100 & 57.19 & 66.59 & 131.4 & 57.12 & 65.52 & 131.33 \\
\hline 250 & 65.29 & 76.7 & 139.5 & 64.22 & 77.63 & 134.43 \\
\hline 300 & & 78.40 & 141.2 & 65.92 & 78.13 & 135.13 \\
\hline
\end{tabular}

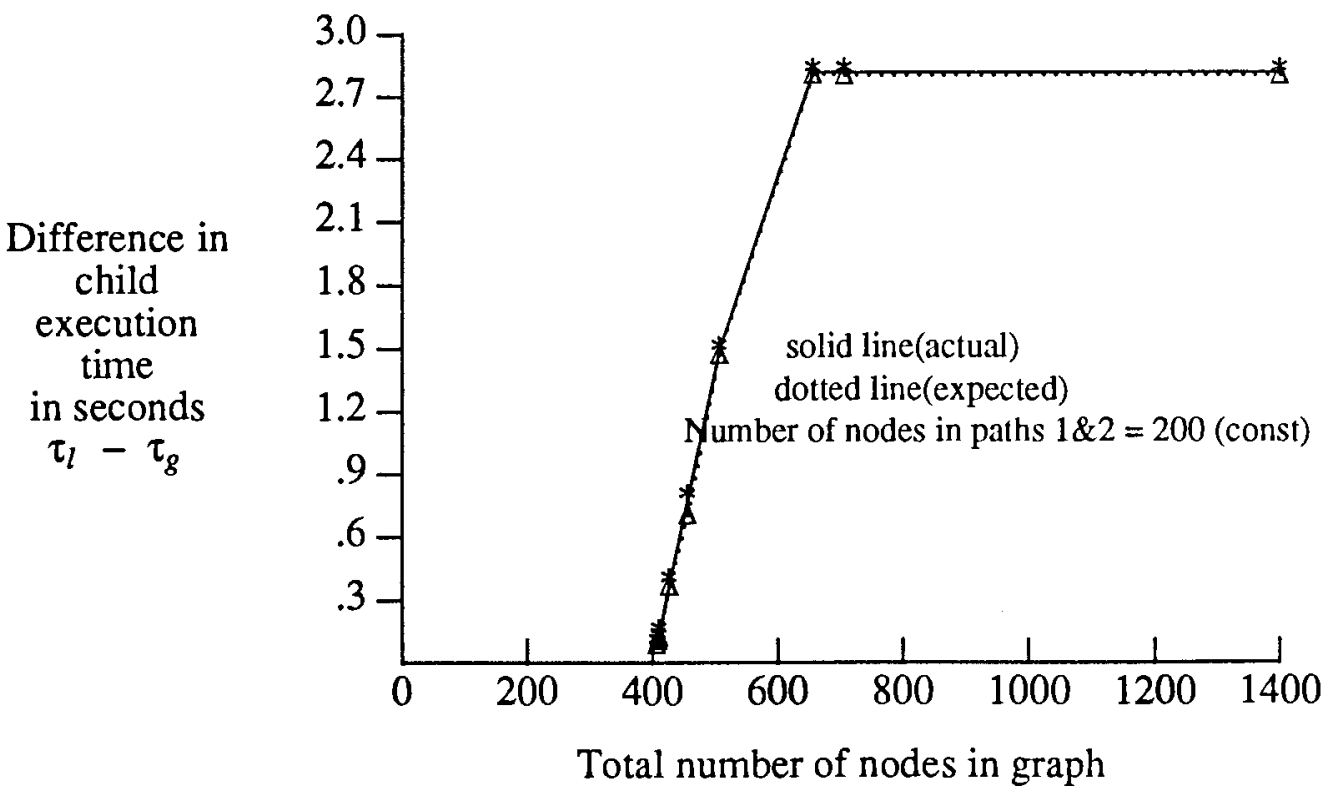

Figure 18.Graph for Variation in Number of Nodes on Path 3

Nodes on Paths $1 \& 2=200$. 


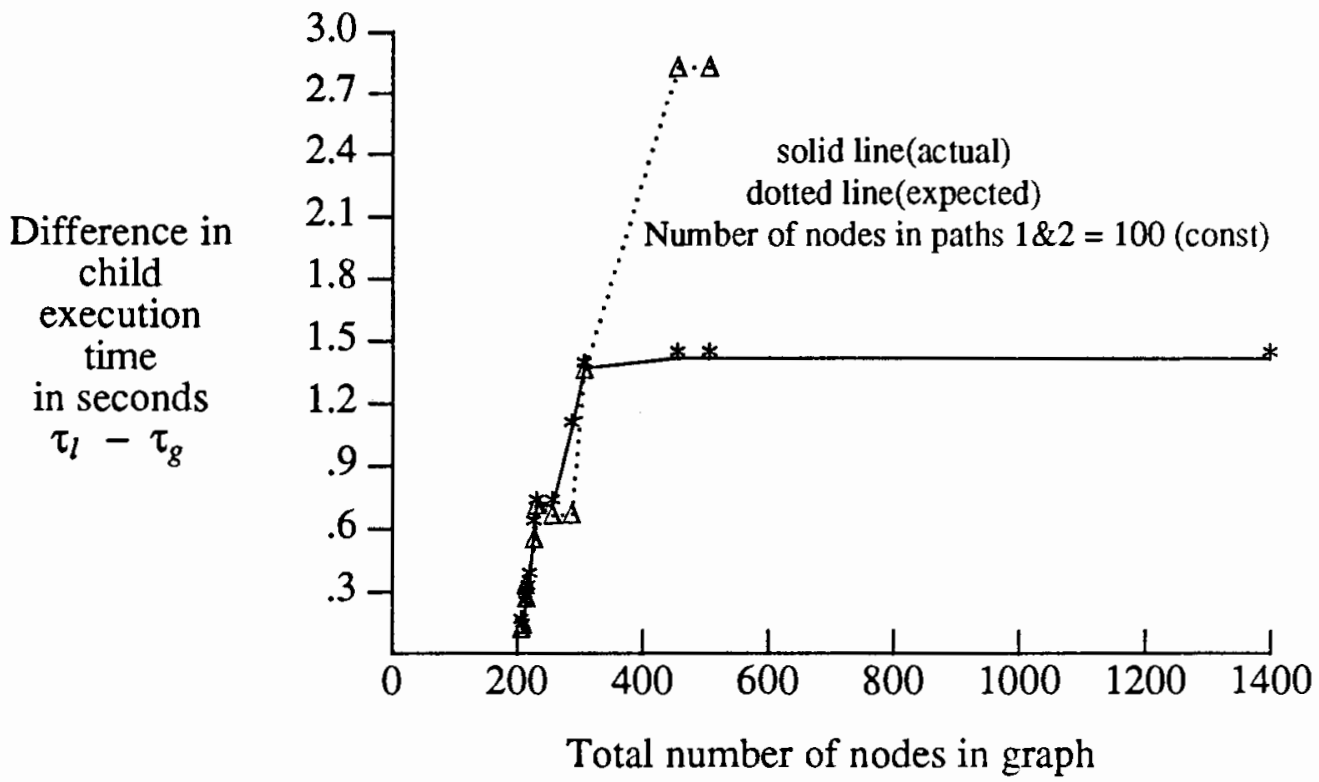

Figure 19.Graph for Variation in Number of Nodes on Path 3 Nodes on Paths $1 \& 2=100$.

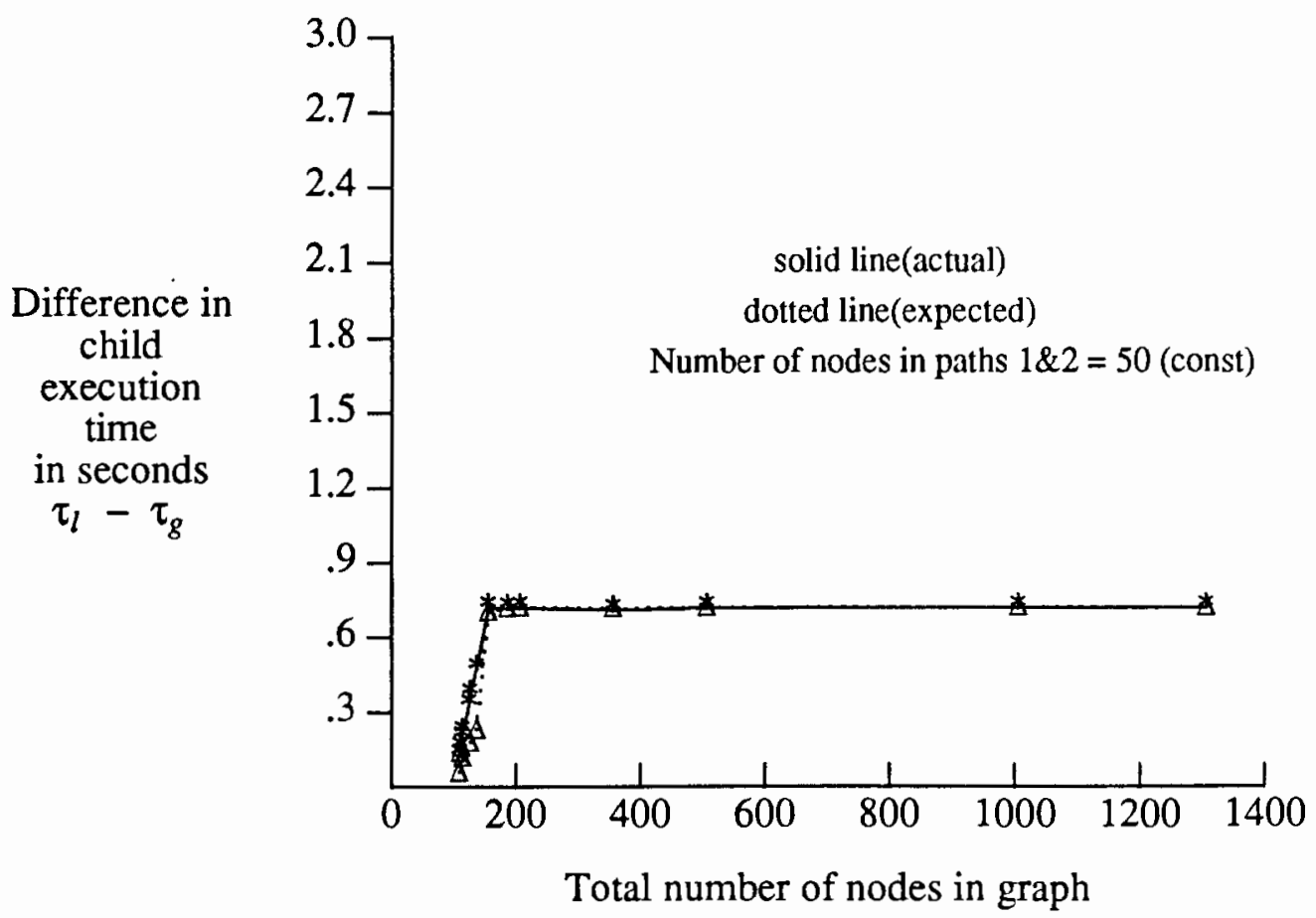

Figure 20.Graph for Variation in Number of Nodes on Path 3 Nodes on Paths $1 \& 2=50$. 


\subsection{VARIATION IN PATH 1 (PATH 2 AND 3 CONSTANT)}

Tests were run for nodes in the path 1 ranging from 1 to 1000 . These tests were repeated for nodes in path 2 varying from 50 to 200 while keeping nodes in path 3 constant to 10. The data generated for them is shown in the Tables V and VI under the columns with headings 50,100 and 200. Figures 21,22 and 23 show the difference in actual and expected child execution times for the globally and locally scheduled graph. As per equations 4 and 5 of section 4.3 .3 one would expect that as the number of nodes in path 1 are increased, the number of overlapping nodes in path 1 with that in path 3 would increase linearly and then become a constant. Though this is reflected in the experimental results shown in Tables $\mathrm{V}$ and VI, the difference in execution time does not become a constant once the number of overlapping nodes are constant. The execution time as seen in Figures 21, 22 and 23 varies with variation in the number of nodes in path 2. The reason for such a behavior, is that, as the number of nodes in path 1 increases they are assigned higher and higher priority than those in path 2 . This results in some of the nodes in path 1 firing alternately with the nodes in path 2 . Since this sequence of firing of nodes is similar to that usually observed in the local schedule case, the execution time of the global schedule slowly starts approaching that of local schedule and the difference between them decreases. 
TABLE $V$

EXECUTION TIME FOR GRAPHS WITH PATH 1 VARYING (GLOBAL SCHEDULE)

\begin{tabular}{|r|c|c|r|r|r|r|r|r|r|}
\hline \multirow{2}{*}{$\begin{array}{c}\text { Nodes in } \\
\text { path 3 }\end{array}$} & \multicolumn{3}{|c|}{$\begin{array}{c}\text { Child execution time } \\
\text { (measured in sec.) }\end{array}$} & \multicolumn{3}{c|}{$\begin{array}{c}\text { Overlapping nodes } \\
\text { in path 1 }\end{array}$} & \multicolumn{3}{|c|}{$\begin{array}{c}\text { Child execution time } \\
\text { (expected in sec.) }\end{array}$} \\
\cline { 2 - 11 } & 50 & 100 & 200 & 50 & 100 & 200 & \multicolumn{1}{|c|}{50} & 100 & 200 \\
\hline 1 & 0.90 & 2.69 & 2.06 & 2 & 1 & 2 & 0.65 & 1.33 & 2.03 \\
\hline 5 & 1.32 & & 3.08 & 5 & 6 & 4 & 0.89 & 1.36 & 2.63 \\
\hline 10 & 1.39 & 1.37 & 4.15 & 11 & 11 & 12 & 1.16 & 2.62 & 3.59 \\
\hline 50 & 1.94 & 2.99 & 4.67 & 13 & 17 & 16 & 1.68 & 2.39 & 3.99 \\
\hline 100 & 2.21 & 3.65 & 8.01 & 16 & 15 & 11 & 2.11 & 3.83 & 7.57 \\
\hline 300 & 5.88 & 6.93 & 11.39 & 14 & 15 & 15 & 5.03 & 6.52 & 10.56 \\
\hline 500 & 8.69 & 10.30 & 19.30 & 16 & 16 & 14 & 8.30 & 9.52 & 17.81 \\
\hline
\end{tabular}

TABLE VI

EXECUTION TIME FOR GRAPHS WITH PATH 1 VARYING (LOCAL SCHEDULE)

\begin{tabular}{|c|c|c|c|c|c|c|}
\hline \multirow{2}{*}{$\begin{array}{c}\text { Nodes in } \\
\text { path 3 }\end{array}$} & \multicolumn{3}{|c|}{$\begin{array}{c}\text { Wall clock time } \\
\text { (measured in sec.) }\end{array}$} & \multicolumn{3}{c|}{$\begin{array}{c}\text { Wall clock time } \\
\text { (expected in sec.) }\end{array}$} \\
\cline { 2 - 7 } & 50 & 100 & 200 & 50 & 100 & 200 \\
\hline 1 & 0.90 & 2.69 & 2.08 & 0.65 & 1.36 & 2.03 \\
\hline 5 & & & 3.16 & 0.89 & 1.49 & 2.54 \\
\hline 10 & 1.39 & 1.52 & 4.30 & 1.16 & 1.81 & 3.43 \\
\hline 50 & 1.96 & 3.59 & 5.14 & 1.70 & 2.87 & 4.47 \\
\hline 100 & 2.24 & 4.12 & 8.48 & 2.08 & 4.30 & 8.05 \\
\hline 300 & 6.91 & 7.14 & 11.78 & 5.06 & 6.73 & 10.95 \\
\hline 500 & 8.72 & 19.59 & 8.33 & 10.32 & 18.10 & \\
\hline
\end{tabular}


Difference in child execution time
in seconds
$\tau_{l}-\tau_{g}$

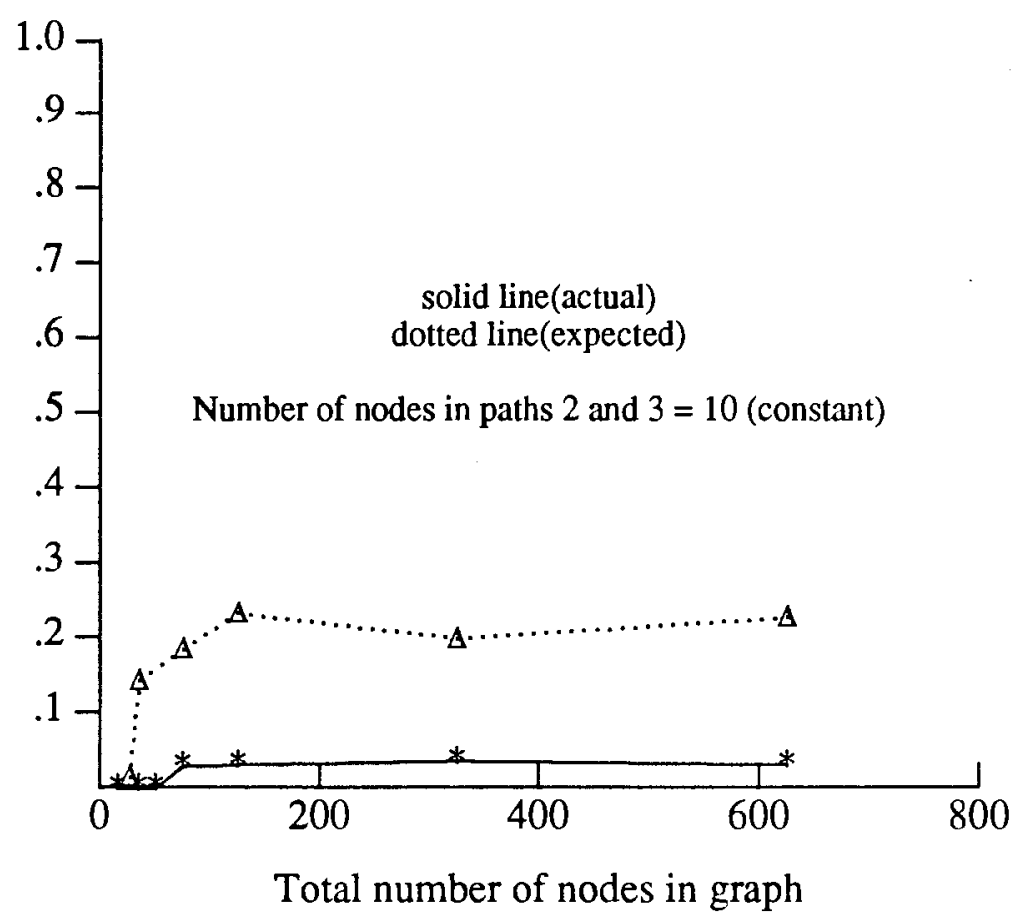

Figure 21.Graph for Variation in Number of Nodes on Path1 Nodes on Paths $2 \& 3=10$.

Difference in child execution time
in seconds $\tau_{l}-\tau_{g}$

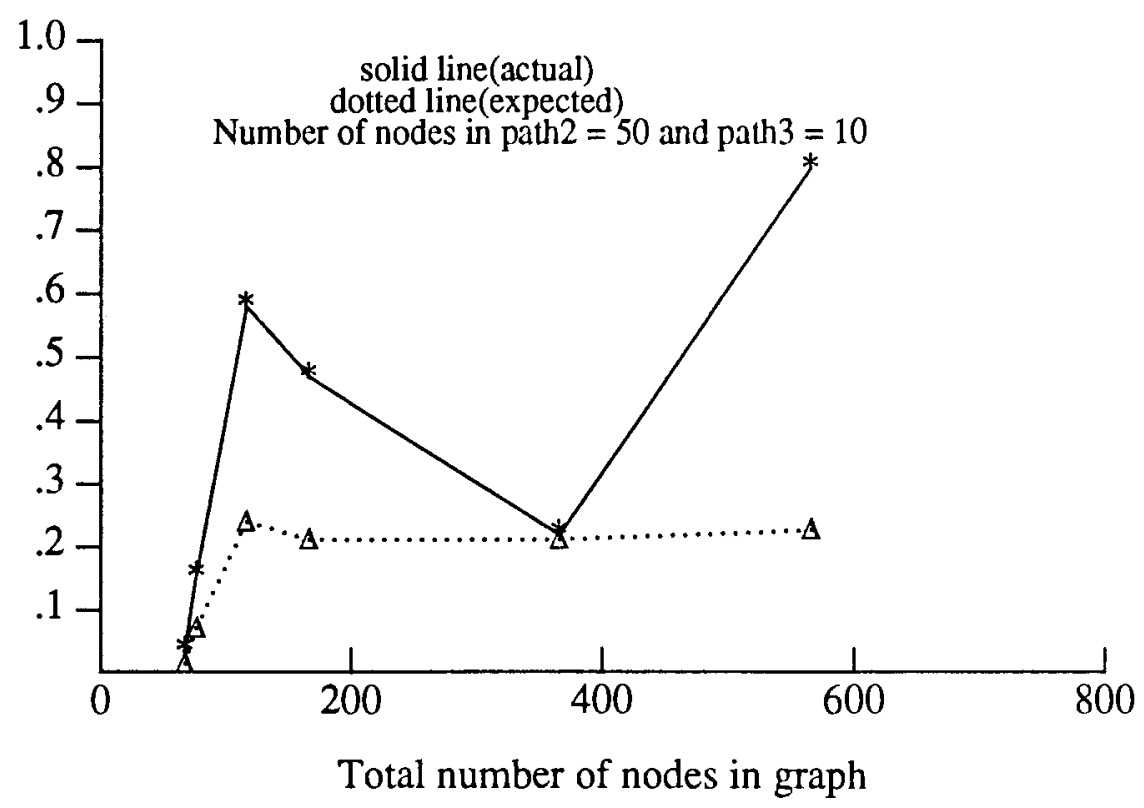

Figure 22.Graph for Variation in Number of Nodes on Path1 Nodes on Path $2=50 \&$ Path $3=10$. 


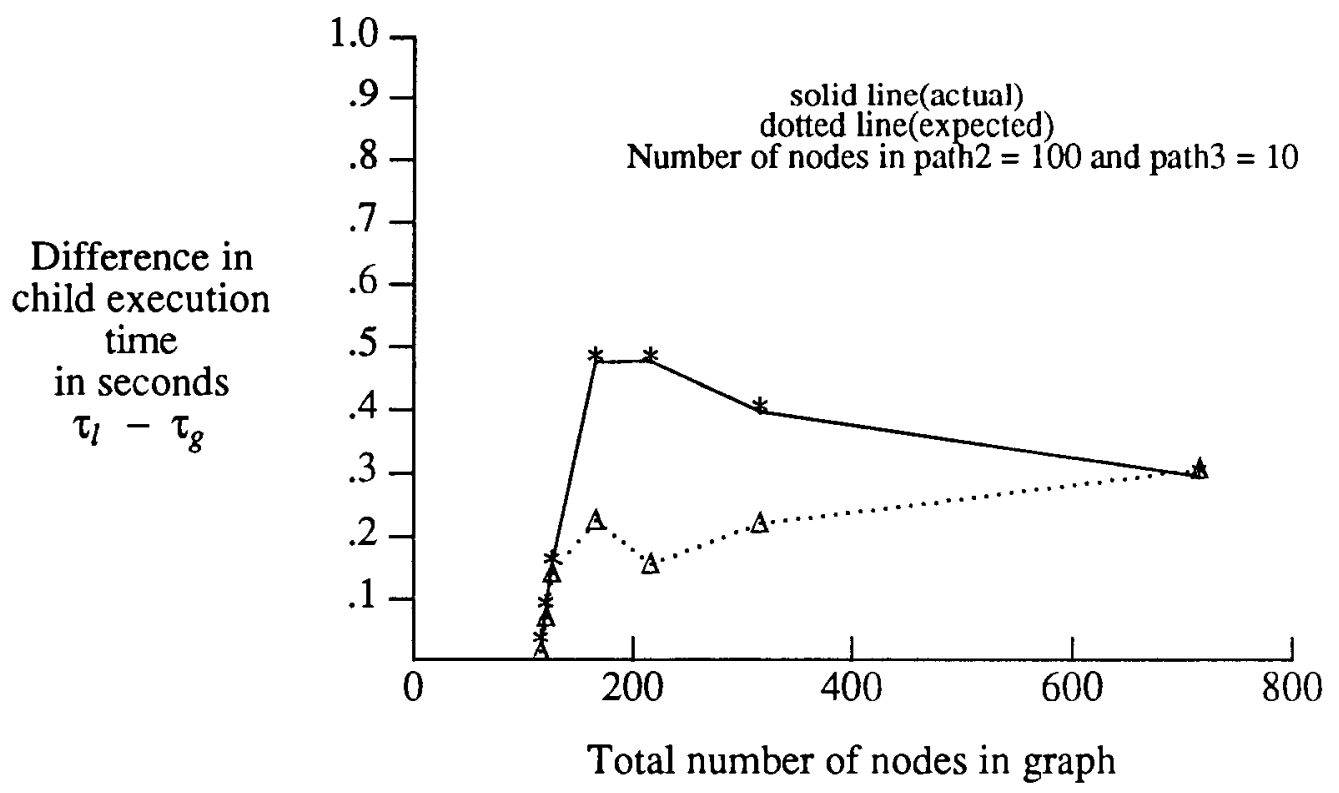

Figure 23.Graph for Variation in Number of Nodes on Path1 Nodes on Path $2=100 \&$ Path $3=10$.

\subsection{VARIATION IN PATH 2 (PATH 1 AND 3 CONSTANT)}

Tests were run for nodes in path 2 ranging from 1 to 1000 . These tests were repeated for nodes in path 1 varying from 10 to 100 while keeping nodes in path 3 constant to 10. The data generated for them is shown in Tables VII and VIII under the columns with headings 10,50 and 100 . Figures 24 and 25 show the difference in actual and expected child execution times. The results of simulation show that the difference in execution time when nodes in path 1 are 50 and 100 respectively, is erratic and dips steeply at certain points. The difference however increases linearly when the nodes in path 1 equal 10 and follows the results expected from equations 9 and 11 in Chapter 4.3.4.

The reason for a non-linear increase in the execution time difference when the number of nodes in path 1 are 50 and 100 is, a variation in the inter-processor communication, which is reflected as a sudden increase in the number of overlapping nodes in path 1 in Tables VII and VIII. 
TABLE VII

EXECUTION TIME FOR GRAPHS WITH PATH 2 VARYING (GLOBAL SCHEDULE)

\begin{tabular}{|c|c|c|c|c|c|r|r|r|r|}
\hline \multirow{2}{*}{$\begin{array}{c}\text { Nodes in } \\
\text { path 3 }\end{array}$} & \multicolumn{3}{|c|}{$\begin{array}{c}\text { Wall clock time } \\
\text { (measured in sec.) }\end{array}$} & \multicolumn{3}{c|}{$\begin{array}{c}\text { Overlapping nodes } \\
\text { in path 1 }\end{array}$} & \multicolumn{3}{c|}{$\begin{array}{c}\text { Wall clock time } \\
\text { (expected in sec.) }\end{array}$} \\
\cline { 2 - 11 } & 50 & 100 & 200 & 50 & 100 & 200 & \multicolumn{1}{c|}{50} & 100 & \multicolumn{1}{c|}{200} \\
\hline 1 & 0.91 & 1.65 & 3.99 & 6 & 17 & 9 & 0.65 & 1.81 & 3.98 \\
\hline 5 & 0.99 & 2.17 & 5.12 & 11 & 11 & 8 & 0.65 & 1.83 & 4.00 \\
\hline 10 & 0.57 & 2.39 & 4.67 & 11 & 10 & 9 & 0.65 & 1.86 & 4.11 \\
\hline 50 & 2.31 & 1.97 & 4.36 & 11 & 13 & 16 & 1.98 & 1.67 & 4.19 \\
\hline 100 & 1.87 & 2.67 & 7.37 & 10 & 10 & 15 & 1.99 & 2.03 & 7.23 \\
\hline 400 & 8.31 & 9.43 & 11.54 & 11 & 13 & 15 & & 9.51 & 11.42 \\
\hline 600 & 10.66 & 12.21 & & 11 & 11 & 14 & 9.31 & 11.42 & 16.43 \\
\hline 800 & 15.54 & 16.66 & 19.14 & 13 & 12 & 18 & 15.31 & 16.59 & 18.02 \\
\hline
\end{tabular}

TABLE VIII

EXECUTION TIME FOR GRAPHS WITH PATH 2 VARYING (LOCAL SCHEDULE)

\begin{tabular}{|c|c|c|c|c|c|c|}
\hline \multirow{2}{*}{$\begin{array}{c}\text { Nodes in } \\
\text { path 3 }\end{array}$} & \multicolumn{3}{|c|}{$\begin{array}{c}\text { Wall clock time } \\
\text { (measured in sec.) }\end{array}$} & \multicolumn{3}{c|}{$\begin{array}{c}\text { Wall clock time } \\
\text { (expected in sec.) }\end{array}$} \\
\cline { 2 - 7 } & 50 & 100 & 200 & 50 & 100 & 200 \\
\hline 1 & 1.21 & 1.78 & 4.37 & 0.65 & 1.91 & 3.85 \\
\hline 5 & 1.21 & 2.03 & 5.77 & 0.68 & 1.99 & 4.03 \\
\hline 10 & 2.37 & 2.44 & 4.69 & 0.71 & 2.23 & 4.22 \\
\hline 50 & 2.36 & 2.01 & 4.91 & 2.04 & 1.75 & 4.61 \\
\hline 100 & 1.99 & 2.39 & 7.79 & 1.91 & 2.05 & 7.45 \\
\hline 400 & 9.36 & 9.46 & 11.52 & & 10.04 & 11.81 \\
\hline 600 & 11.05 & 12.32 & & 10.03 & 11.65 & 17.04 \\
\hline 800 & 16.02 & 16.99 & 20.22 & 16.11 & 17.59 & 18.32 \\
\hline
\end{tabular}




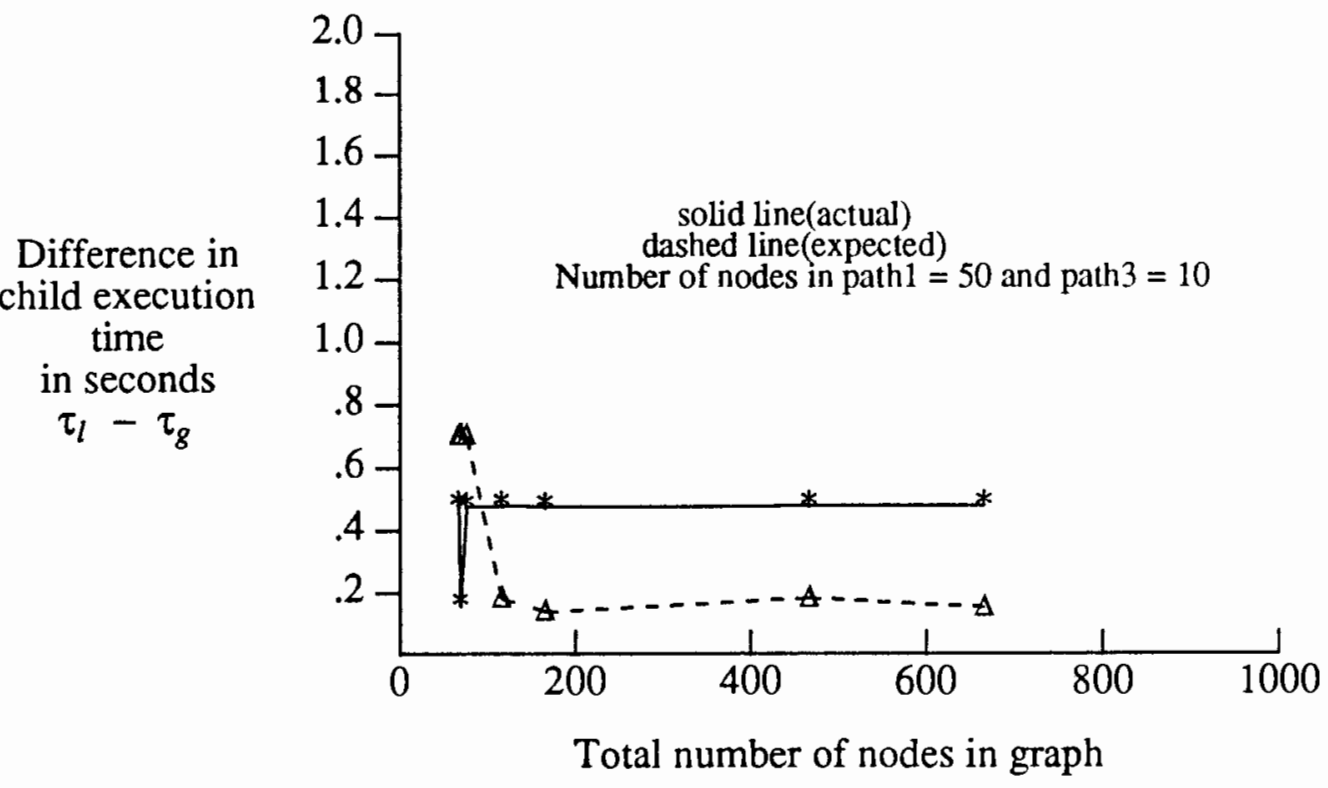

Figure 24.Graph for Variation in Number of Nodes on Path2 Nodes on Path $1=50 \&$ Path $3=10$.

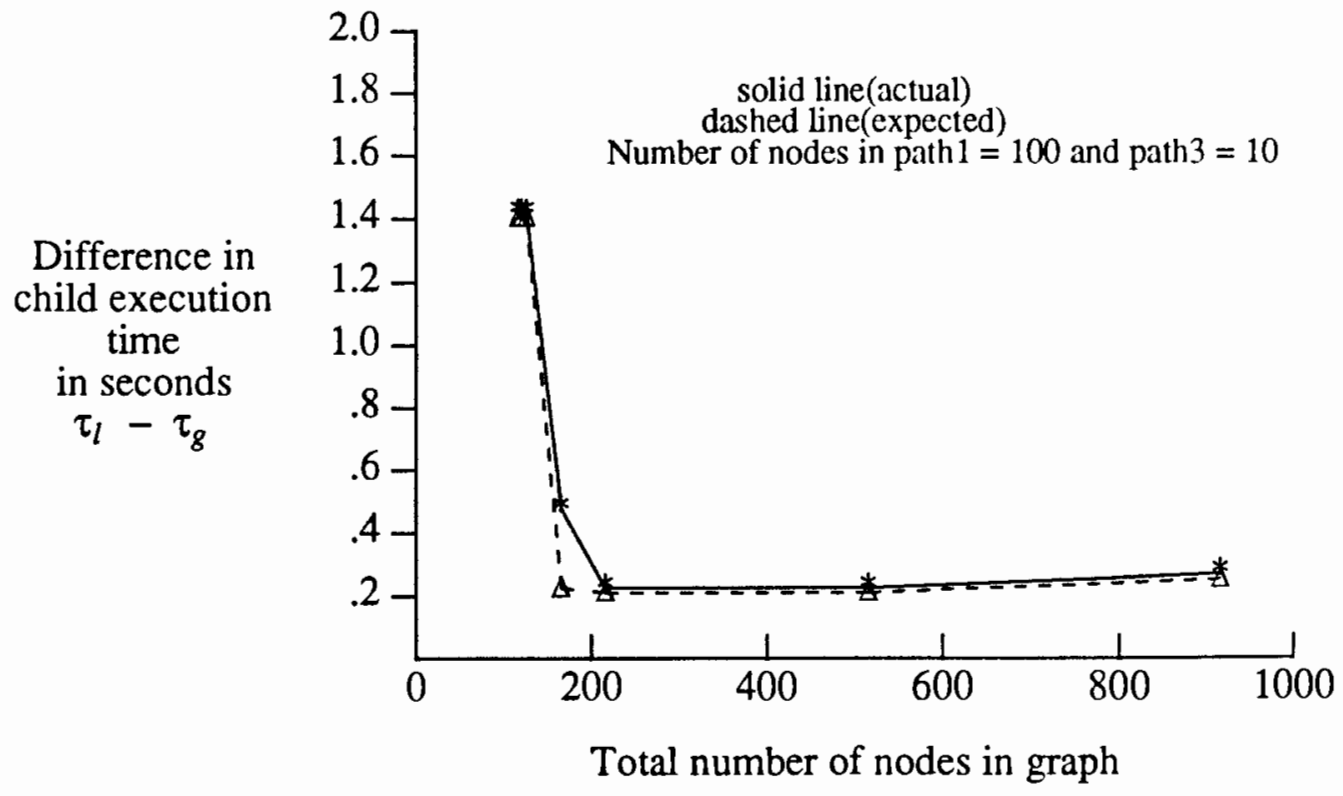

Figure 25.Graph for Variation in Number of Nodes on Path2 Nodes on Path $1=100$ and Path $3=10$. 


\subsection{MODEL PERFORMANCE ANALYSIS}

The results of experiments closely follow the predictions made by the models. Even though an effort was made to get a close match between the measured and expected values by averaging the measured values over 20 iterations, a big difference in the expected and measured values was found at times and is reflected in the tables. This difference is a result of a variation in setup communication time because of changes in the load over the network. As seen in most of the Figures, the difference in the execution time of globally and locally scheduled graphs is not significant and as seen in Figure 26 the execution time of the global scheduler is far more than that of the local scheduler. It can be clearly concluded from the graph that the local schedule is cheaper in terms of time to order a set of nodes at a processor. These results strongly support our hypothesis that the interprocessor communication can be ignored during ordering of operations at a processor.

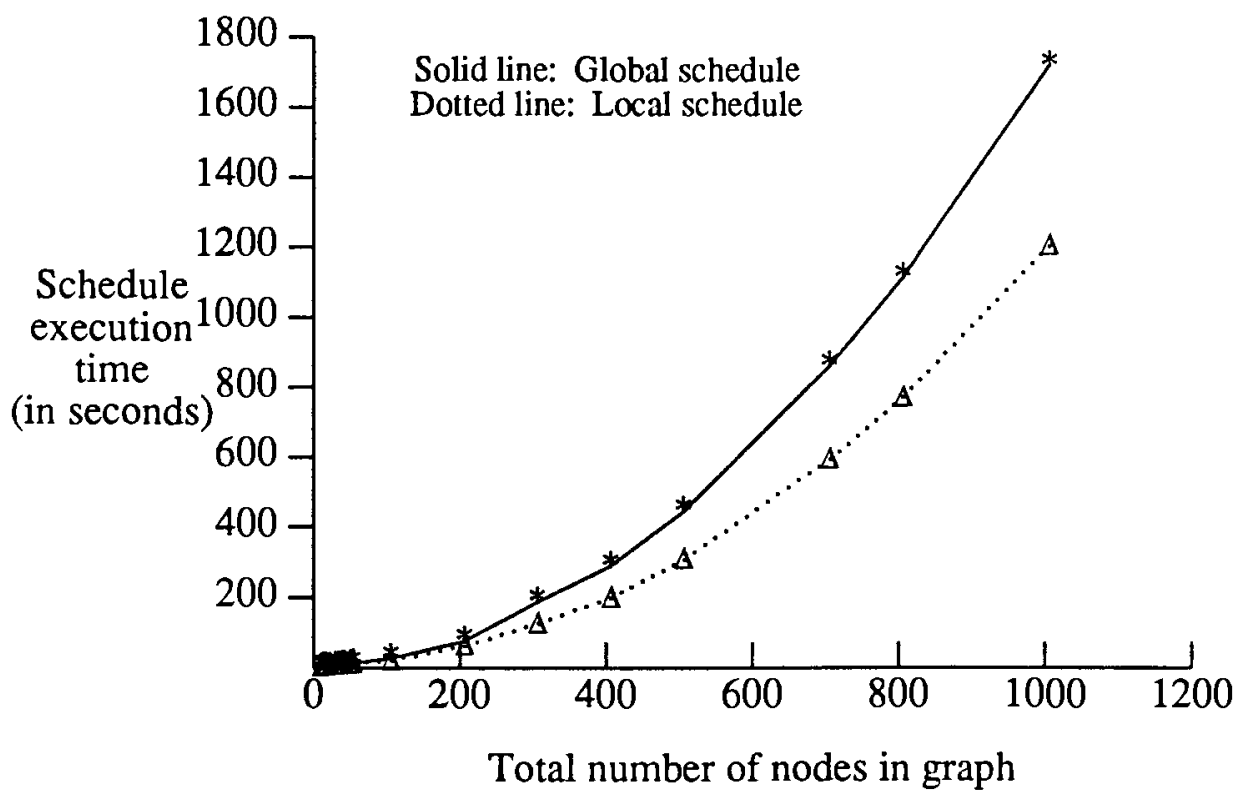

Figure 26.A Comparison of Global and Local Schedular Execution times. 


\section{CHAPTER VI}

\section{CONCLUSION}

\subsection{SIGNIFICANCE OF THIS RESEARCH}

This thesis presented an analysis of ignoring inter-processor communication during intraprocessor scheduling. Scheduling in our sense is different than that suggested in most of the literature available in that it does not include allocation. To aid this research two scheduling techniques, namely, global and local, have been suggested in Chapter II. Intraprocessor scheduling is important because it can take advantage of parallelism among nodes in a given task at a processor, and order the execution sequence of those nodes at the processor. This ordering can result in a reduced execution time of the subgraph, depending on the efficiency of the scheduling algorithm used. Eight scheduling heuristics have been implemented in the ParPlum system. However, Granski's scheduling algorithm is the most effective among them and has been used for evaluating the global and local scheduling techniques. To test the techniques a graph was sought after, which identified some properties that are necessary to see a difference in global and local schedules. For fork and join graphs, the key property is: A path in a program has to be cut and its pieces assigned to different processors to see a difference in relative priorities.

An analysis of the results suggests that the difference between globally and locally scheduled graphs is visible only when a graph depicts the property mentioned above and when communication time is larger than the execution time of a node. In other cases the models and experimental results show no difference in the execution time of 
globally and locally scheduled graphs. Figure 26 , on the other hand, suggests that the local scheduler is cheaper than the global scheduler in terms of execution time. Considering the high cost of global schedule execution time and its almost negligible difference with the local schedule time, it can be suggested that, one can ignore inter-processor communication while ordering operations at a processor. Since the allocation process is sensitive to IPC [9], it seems that allocation must pay attention to IPC, while scheduling can ignore it.

\subsection{FUTURE WORK}

Granski [13] has suggested that scheduling is an expensive overhead in the mapping process and that adding a priority mechanism is not justifiable in the general case because of implementation restrictions in a dataflow computer. It would be interesting to analyze scheduling of operations at a processor on a coarse grain basis. For example, let us consider scheduling of operations in the graph of Figure 27.

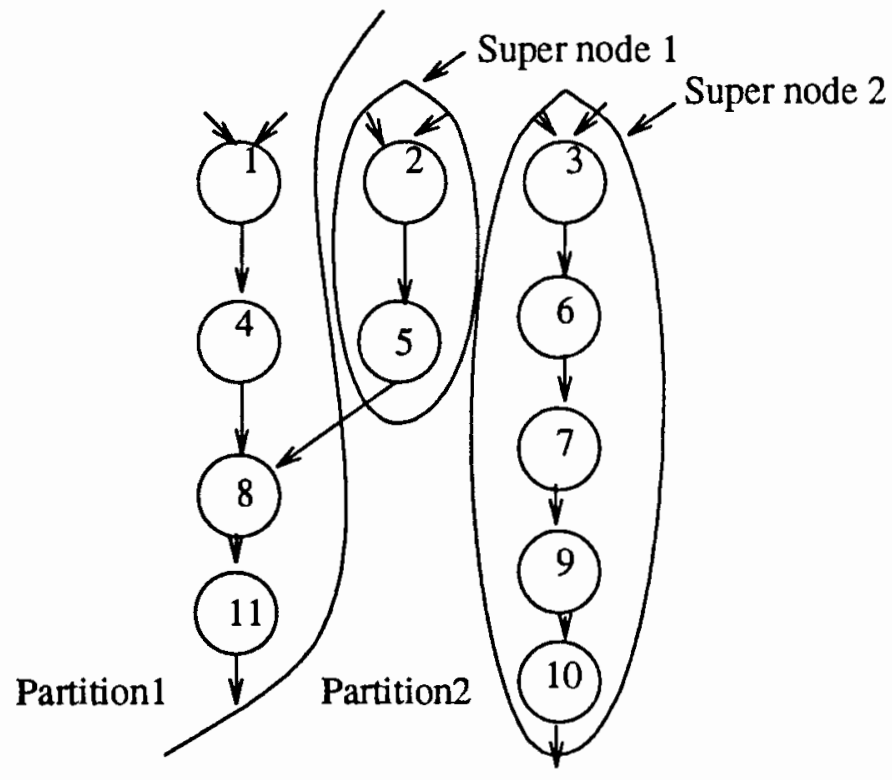

Figure 27.An example of Fine and Coarse Grain Scheduling. 
Assuming that strings 1 and 2 of nodes in partition 2 can be compounded to represent two super nodes, let us call ordering of the super nodes Coarse Grain scheduling. If communication time among processors is the same as the execution time of a node $(c=t=.1)$, the following assignment of priorities would result:

\section{TABLE IX}

\section{SCHEDULE FOR FINE GRAIN}

\begin{tabular}{|c|c|c|c|c|}
\hline \multirow{2}{*}{ Actor\# } & \multicolumn{2}{|c|}{ global } & \multicolumn{2}{c|}{ local } \\
\cline { 2 - 5 } & weight & priority & weight & priority \\
\hline 1 & .4 & 1 & .4 & 1 \\
\hline 2 & .5 & 1 & .2 & 4 \\
\hline 3 & .5 & 1 & .5 & 1 \\
\hline 4 & .3 & 2 & .3 & 2 \\
\hline 5 & .4 & 2 & .1 & 5 \\
\hline 6 & .4 & 2 & .4 & 2 \\
\hline 7 & .3 & 3 & .3 & 3 \\
\hline 8 & .2 & 3 & .2 & 3 \\
\hline 9 & .2 & 4 & .2 & 4 \\
\hline 10 & .1 & 5 & .1 & 5 \\
\hline 11 & .1 & 4 & .1 & 4 \\
\hline
\end{tabular}

In the case of coarse grain scheduling a similar assignment of weights would result, but super node 1 would be assigned the higher priority and all nodes in it will be executed before those in super node 2. The equations for the execution time of the two scheduling cases would then be:

Global schedule( Fine grain) :

$$
t^{2,1}+t^{3,4}+c_{5,8}^{6}+t^{8,7}+t^{11,9}+t^{10}=6 t+c
$$

Local schedule( Fine grain) :

$$
t^{3,1}+t^{6,4}+t^{7}+t^{2}+t^{9}+t^{5}+c_{5,8}^{10}+t^{8}+t^{11}=8 t+c
$$

Global schedule( Coarse grain) :

$$
t^{2,1}+t^{5,4}+c_{5,8}^{3}+t^{8,6}+t^{11,7}+t^{9}+t^{10}=6 t+c
$$


Local schedule( Coarse grain) :

$$
t^{3,1}+t^{6,4}+t^{7}+t^{9}+t^{10}+t^{2}+t^{5}+c_{5,8}+t^{8}+t^{11}=9 t+c
$$

The equation for the coarse grain local schedule shows a smaller difference in execution time than those in the fine grain case. Since a coarse grain schedule would take shorter time to produce than a fine grain schedule, one would obviously choose the former while sacrificing the small difference in execution time. This is an interesting property and could be studied further.

All analysis in this thesis assumed that a processor could have only one ALU. If, however, the number of ALU's available in each processor grows to more than the number of ready operations at that processor, then there would be no need to schedule the operations. On the contrary, if the number of available ALU's is less than the operations, one feels a strict need of scheduling. The performance of parallel execution is therefore a function of the number of available ALU's. This is an interesting topic and can be studied further.

All of the graphs tested for this thesis had nodes of the same datatype. This notion, though convenient for our testing, is not mandatory and graphs having nodes of different data types should be executed for global and local schedules. For our experimental purpose we chose a very simple graph. However, more complex graphs should be tested. Two models have been extensively used in estimating the execution time of a node and the communication time between processors in Granski's algorithm and also in the equations for global and local schedules shown in Chapter IV. The behavior of global and local schedules towards inaccuracies in those models would be worth looking into. To avoid the variations in the parameters because of changes in the load average on the system, the simulator could be implemented on a dedicated multiprocessor, such as Intel's Hypercube. 


\section{REFERENCES}

[1] Adam, T. L., K. M. Chandy, and J. R. Dickson, "A Comparison of List Schedules for Parallel Processing Systems," Communications ACM, vol. 17, no. 12, pp. 685-690, December 12, 1974.

[2] Anger, F. D., J. Hwang, and Y. Chow, "Scheduling with Sufficiently Loosely Coupled Processors," Journal of Parallel and Distributed Computing, vol. 9, pp. 87-92, 1990.

[3] Appelbe, W. F. and M. R. Ito, "Scheduling Heuristics in a Multiprogramming Environment," IEEE Transactions on Computers, vol. c-27, no. 7, pp. 628-637, July 1978.

[4] Coffman, E. G., "Computer and Job-Shop Scheduling Theory," John Wiley \& Sons, New York , 1976.

[5] Driscoll, M. A., P. R. Prins, P. D. Fisher, and L. M. Ni, "Efficient Scheduling of Dataflow Graphs for Multiprocessor Architectures," Rep. no. MSU-ENGR-88008, College of Engineering, Michigan State University June, 1988.

[6] Driscoll, M. A. and P. D. Fisher, "On Partitioning of Algorithms for Parallel Execution on VLSI Circuit Architectures," Rep. no. MSU-ENGR-88-016, College of Engineering, Michigan State University , September, 1988.

[7] Driscoll, M. A., J. Fu, S. M. Pai, C. Patwardhan, L. Setiowijoso, D. Tang, and K. Thayib, "Sensitivity Analysis and Mapping Programs to Parallel Architectures," Presented at the 1991 International Conference on Parallel Processing , August 1991.

[8] Efe, K., "Heuristic Models of Task Assignment Scheduling in Distributed Systems," Computer, vol. 15, pp. 50-56, June 1982.

[9] El-Rewini, H. and T. G. Lewis, "Scheduling Parallel Program Tasks onto Arbitrary Target Machines," Journal of Parallel and Distributed Computing , vol. 9, pp. 138-158, 1990.

[10] Fu, J., "PARPLUM A System for Evaluating Parallel Program Optimization Methods," M.S Thesis, Department of Electrical Engineering, Portland State University , 1991. 
[11] Gonzalez, M. J. Jr., "Deterministic Processor Scheduling," Computing Surveys, vol. 9, no. 3, pp. 173-204, September 1977.

[12] Graham, R. L., "Bounds on Multiprocessor Timing Anomalies," SIAM J. Appl. Math. , vol. 17, pp. 416-429, 1969.

[13] Granski, M., "The Effect of Operation Scheduling on the Performance of a Data Flow Computer," IEEE Transaction on Computers, vol. C-36 no. 9, September 1987.

[14] Ha, S. and E. A. Lee, "Compile Time Scheduling and Assignment of Data-FLow Graphs with Data-Dependent Iteration," IEEE Transaction on Computers, vol. 40, no. 11, Nov. 1991.

[15] Hu, T. C., "Parallel Sequencing and Assembly Line Problems," Oper. Res. , vol. 9, 841-848, November 1961.

[16] Hwang, J., Y. Chow, F. D. Anger, and C. Lee, "Scheduling Precedence graphs in Systems with Interprocessor Communication Times," Siam J. Computing, vol. 18, no. 2, pp. 244-257, April 1989.

[17] Jordan, H., "The Characteristics of Parallel Algorithms," Chapter 16 MIT Press, Cambridge, MA, 1987.

[18] Karp, A., "Programming for Parallelism," Computer , vol. 15, pp. 43-57, May 1987.

[19] Kasahara, H. and S. Narita, "Practical Multiprocesor Scheduling Algorithm for Efficient Parallel Processing," IEEE Transactions on Computer, vol. c-33, no. 11, pp. 1023-1029, November 1984.

[20] Kaufman, M. T., "An Almost-Optimal Algorithm For The Assembly Line Scheduling Problem," IEEE Transactions on Computers, vol. C-23, 1169-1174, Nov. 1974.

[21] Kohler, W. H., "A Preliminary Evaluation of the Critical Path Method for Scheduling Tasks on Multiprocessor Systems," IEEE Transactions on Computers , vol. c-15, no. 12, pp. 1235-1238, December 1975.

[22] Kruatrachue, B., "Static Task Scheduling and Grain Packing in Parallel Processing Systems," Ph.D Thesis, Department of Computer Science, Oregon State University, 1987.

[23] Kunde, M., "Nonpreemptive LP-Scheduling on Homogeneous Multiprocessor Systems," SIAM J. Computing , vol. 10, no. 1, pp. 151-173, Feb. 1981. 
[24] Lenstra, J. K. and A. H. G. Rinnooy Kan, "Complexity Of Scheduling Under Precedence Constraints," Operations Research, vol. 26, no. 1, pp. 22-35, Jan.-Feb. 1978.

[25] Li, Hon F., "Scheduling Trees in Parallel/Pipelined Processing Environments," IEEE Transactions on Computers, vol. c-26, no. 11, pp. 1101-1112, November 1977.

[26] Martin, D. and G. Estrin, "Models of Computation and System Cyclic to Acyclic Graph Transformations," IEEE Transaction Elec. Comput. , vol. C-16, pp. 70-79, Feb. 1967.

[27] Papadimitriou, C. H. and J. D. Ullman, "A Communication-Time Tradeoff," SIAM J. Comput. , vol. 6, no. 4, pp. 639-646, Aug. 1987.

[28] Polychronopoulos, C. D. and D. J. Kuck, "Guided Self-Scheduling: A Practical Scheduling Scheme for Parallel Supercomputers," IEEE Transaction on Computers , vol. C-36, no. 12, pp. 1425-1439, December 1987.

[29] Ramamoorthy, C. , K. M. Chandy and M. J. Gonzales, "Optimal Scheduling Strategies in a Multiprocessor System," IEEE Transaction Computer, vol. C-21, pp. 37-146, Feb. 1972.

[30] Ramamoorthy, C. and W. H. Leung, "A Scheme for Parallel Execution of Sequential Programs," Proc. of 1976 International conference on Parallel Processing, pp. 312-316, 1976.

[31] Rayward-Smith, V. J., UET "Scheduling with Interprocessor Communication Delays," Internal Report SYS-C86-06, School of Information Systems, University of East Anglia, Norwich, United Kingdom, 1986

[32] Sarkar, V., "Partitioning and Scheduling Parallel Programs for Multiprocessors," The MIT Press, 1989.

[33] Sethi, R. and Ravi, "Scheduling Graphs on Two Processors," SIAM J. Comput , vol. 5, no. 1, pp. 73-82, March 1976.

[34] Simmons, M., R. Koskela, and I. Bucher, "Instrumentation for Future Parallel Computing Systems," Addison Wesley Publishing Company , 1989. 